\title{
Synthesis of New Trihalomethylated and Non-symmetrical Substituted 2-(1H-Pyrazolyl)-5-(1H-pyrazolylcarbonyl)pyridines
}

\author{
Helio G. Bonacorso,* Gisele R. Paim, Carolina Z. Guerra, Ronan C. Sehnem, Cleber A. Cechinel, \\ Liliane M. F. Porte, Marcos A. P. Martins and Nilo Zanatta
}

Departamento de Química, Universidade Federal de Santa Maria, 97105-900 Santa Maria-RS, Brazil

\begin{abstract}
Este trabalho apresenta a síntese de uma nova série de 2-[3-alquil (aril/heteroaril)-5-trialometil5-hidróxi-4,5-diidro-1H-pirazol-1-il]-5-[3-alquil(aril/heteroaril)-5-trialometil-5-hidróxi-4,5diidro-1H-pirazol-1-il-1-carbonil]piridinas, obtida a partir de reações de ciclocondensação de 4-alcóxi-4-alquil(aril/heteroaril)-1,1,1-trialo-3-alquen-2-onas $\left[\mathrm{CX}_{3} \mathrm{C}(\mathrm{O}) \mathrm{CH}=\mathrm{CR}^{1} \mathrm{OR}\right.$, onde $\mathrm{R}=\mathrm{Me}, \mathrm{Et} ; \mathrm{R}^{1}=\mathrm{H}, \mathrm{Me}, \mathrm{Ph}, 4-\mathrm{MeOPh}, 4-\mathrm{NO}_{2} \mathrm{Ph}, 4,4^{\prime}$-Bifenil, 1-Naftil, Fur-2-il e Tien-2-il e $\mathrm{X}=\mathrm{F}, \mathrm{Cl}$ ] com hidrato de hidrazida 6-hidrazino-nicotínica. Os melhores rendimentos foram obtidos quando as reações foram executadas em etanol como solvente a $78{ }^{\circ} \mathrm{C}$ por 4 horas $(67-97 \%)$. Em sequência, são descritas as reações de desidratação de 2-(1H-pirazol-1-il)-5-(1H-pirazol1-il-1-carbonil)piridinas. Estas reações foram realizadas em meio piridina / benzeno, na presença de cloreto de tionila e levaram ao isolamento de uma série de 2-[3-alquil(aril/heteroaril)-5-trifluormetil1H-pirazol-1-il]-5-[3-alquil(aril/heteroaril)-5-trifluormetil-1H-pirazol-1-il-1-carbonil]piridinas, com rendimentos de 64 a $86 \%$.
\end{abstract}

This paper describes the synthesis of a new series of 2-[3-alkyl(aryl/heteroaryl)5-trifluoro(chloro)methyl-5-hydroxy-4,5-dihydro-1H-pyrazol-1-yl]-5-[3-alkyl(aryl/heteroaryl)5-trifluoro(chloro)methyl-5-hydroxy-4,5-dihydro-1 $H$-pyrazol-1-yl-1-carbonyl]pyridines by the cyclocondensation reaction of 4-alkoxy-4-alkyl(aryl/heteroaryl)-1,1,1-trifluoro(chloro)-3-alken2-ones $\left[\mathrm{CX}_{3} \mathrm{C}(\mathrm{O}) \mathrm{CH}=\mathrm{CR}^{1} \mathrm{OR}\right.$, where $\mathrm{R}=\mathrm{Me}, \mathrm{Et} ; \mathrm{R}^{1}=\mathrm{H}, \mathrm{Me}, \mathrm{Ph}, 4-\mathrm{MeOPh}, 4-\mathrm{NO}_{2} \mathrm{Ph}$, 4,4'-Biphenyl, 1-Naphthyl, Fur-2-yl, Thien-2-yl and X = F, Cl] with 6-hydrazinonicotinic hydrazide hydrate. Yields of 62 to $97 \%$ were obtained when the reactions were performed in ethanol as solvent at $78{ }^{\circ} \mathrm{C}$ for 4 hours. In a subsequent step, the dehydration reactions of 2-(5-hydroxy-1H-pyrazol1-yl)-5-(5-hydroxy-1H-pyrazol-1-yl-1-carbonyl)pyridines were carried out in pyridine/benzene in the presence of thionyl chloride and led to the isolation of a series of 2- [3-alkyl(aryl/heteroaryl)5-trifluoro(chloro)methyl-1H-pyrazol-1-yl]-5-[3-alkyl(aryl/heteroaryl)-5-trifluoro(chloro)methyl$1 H$-pyrazol-1-yl-1-carbonyl]pyridines, in 64 to $86 \%$ yields.

Keywords: pyrazoles, pyridines, heteropolycycles, cyclocondensation reactions, dehydration

\section{Introduction}

Pyrazoles belong to the heterocycles class of compounds and are known to be important intermediates for the preparation of agrochemicals and pharmaceutical compounds..$^{1,2}$ The pyridine, which also deserves much attention, is not found free in nature, but its derivatives have many chemical applications, mostly in polymers and dyes, as well as in the pharmaceutical area as analgesics, anesthetics and psychopharmacological drugs. ${ }^{3}$

\footnotetext{
*e-mail: heliogb@base.ufsm.br
}

A review of the literature has demonstrated that transition-metal complexes of the planar tridentate ligand terpyridine and its substituted derivatives are severely limited by tedious synthesis., 4

More specifically, 2,6-bis( $N$-pyrazolyl)pyridine analogues have also played a remarkably diverse role as chelating ligands in transition metal chemistry. ${ }^{6}$

Moreover, a review of the literature shows only the synthesis of symmetrical and non-symmetrical 2,6-(disubstituted pyrazolyl)- or 2,6-(dipyrazolylcarbonyl)-pyridines. ${ }^{6-13}$

For example, Jameson and Goldsby, ${ }^{7}$ in 1990, prepared a series of four symmetric 2,6-bis-( $N$-pyrazolyl) pyridines (I) in good yields by the reaction of an excess 
of potassium pyrazolate with 2,6-dihalopyridines in diglyme as solvent. In 2005, Mohlala et al. ${ }^{11}$ synthesized 2,6-(dipyrazolylcarbonyl)pyridines (II) from the reaction of 2,6-dichlorodicarbonylpyridine with a series of 3,5-dialkyl substituted $1 \mathrm{H}$-pyrazoles in the presence of triethylamine.

In the same year, Halcrow ${ }^{12}$ published a review showing the synthesis and the coordination chemistry of 2,6-bis(pyrazolyl)pyridines and related ligands such as versatile terpyridine analogues.

In 2006, 2,6-bis-hydrazinopyridine was prepared and characterized for the first time by Garner and coworkers, ${ }^{6}$ in reactions with 1,3-dicarbonyl compounds, this hydrazinopyridine, was a useful material for the preparation of a wide variety of sterically crowded 2,6-bispyrazolylpyridines (III).

Until now, substituted pyridine analogues at positions 2 and 5 have not been described, nor has a simple procedure been found to obtain non-symmetrical substituted pyridines containing pyrazolinyl, carbonylpyrazolinyl and the respective pyrazole substituents at positions 2 and 5 of the pyridine ring. This makes the investigation of 1,1,1-trihalo-4-alkoxy-4-alkyl(aryl/heteroaryl)-3-alken2-ones in reactions with 6-hydrazinonicotinic hydrazide hydrate to obtain the new trihalomethyl-4,5-dihydropyrazolylpyridines and their subsequent intramolecular dehydration an attractive object of study.

The intramolecular dehydration of 4,5-dihydropyrazoles in an acidic medium using pure sulfuric acid, $\mathrm{P}_{2} \mathrm{O}_{5}$, a mixture of sulfuric acid in boiling acetic acid, acetic acid in boiling ethanol, hydrochloric acid/ethanol, acetic anhydride, or acetic acid has been the most employed method for dehydration. ${ }^{14}$ However, in many cases, acid sensitive groups, such as carbonyl, are lost during the dehydration reaction due to the acidic conditions and temperature. In these cases, it has been necessary to employ thionyl chloride and pyridine in benzene as the dehydation medium to obtain the respective pyrazoles. ${ }^{15}$

Alkylhalogenated substituents, especially fluorine, ${ }^{18}$ have been shown to play an important role in heterocyclic compounds, both in synthetic and chemical intermediates and from the biological point of view, as they present pharmacologic activity. ${ }^{16,17}$ Moreover, trichloromethylated heterocyclic are relatively rare, but show promising biological activity.

\section{Results and Discussion}

Considering the importance of 4,5-dihydro-pyrazoles, $1 H$-pyrazoles, pyridines, and the new and little studied pyrazolylpyridine system, the aim of this study is to report the results of cyclocondensation reactions of 1,1,1-trifluoro(chloro)-4-alkyl(aryl/heteroaryl)-4-alkoxy-3alken-2-ones $(\mathbf{1}, \mathbf{2})^{19-27}$ with 6-hydrazinonicotinic hydrazide hydrate to obtain new trifluoro(chloro)methylated2,5-bis(4,5-dihydro-pyrazolyl)pyridines $(\mathbf{3}, \mathbf{4})$ and subsequent dehydration reactions to obtain the respective trifluoro(chloro)methylated pyrazolylpyridines $(\mathbf{5}, \mathbf{6})$, as three aromatic heterocyles. The method is general and it allows for the convenient synthesis of a series of ligands in which the substituents at positions 3 and 5 of the pyrazole rings can be varied.

A review of the literature shows that the methodologies that have been used until now, though efficient for the synthesis of 2,6-dissubstituted pyridines, require drastic conditions and a long reaction time, varying from 12 hours to 5 days, and also involve many reactions steps and an inert atmosphere. Therefore, we have sought to develop a simple method, where it is possible to obtain new 2,5-dissubstituted pyridines in a one-pot reaction and in good yields.

Pyrazole was chosen as the imine donor to replace pyridine in terpyridine for many reasons. Firstly, pyrazoles are not an effective donor atom for a wide variety of transition metals. The weaker basicity of pyrazoles compared with pyridine and their tendency to bind to metals somewhat more weakly than pyridine should be overcome in this system by the chelate effect. Secondly, the pyrazole ring is regiospecifically prepared from the cyclocondensation reaction of the trihalomethylated vinyl

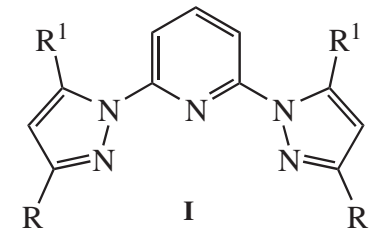

$\mathrm{R}: \mathrm{H}, \mathrm{Me}, \mathrm{Ph}, t$-Bu; $\mathrm{R}^{1}: \mathrm{H}, \mathrm{Me}$

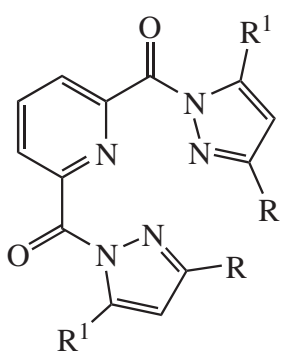

II<smiles>[R]c1cc([R])n(-c2cccc(-n3nc([R])cc3[R])n2)n1</smiles>

$\mathrm{R}: \mathrm{Me}, \mathrm{Ph}, t-\mathrm{Bu}$

$\mathrm{R}=\mathrm{R}^{1}=\mathrm{H}, \mathrm{Me}, t-\mathrm{Bu}$.

Figure 1. Structures of the Bis-pyrazolyl-pyridines. 
ketones $(\mathbf{1}, \mathbf{2})$ with 6-hydrazinonicotinic hydrazide hydrate; and thirdly, until now transition-metal complexes involving these new heterocyclic systems are unknown.

Thus, the reactions of 1,1,1-trifluoro(chloro)-4alkyl(aryl/heteroaryl)-4-alkoxy-3-alken-2-ones (1a-i and 2c-f) ${ }^{19-27}$ with 6-hydrazinonicotinic hydrazide hydrate to obtain the new pyrazolyl-pyridine system $(\mathbf{3}, \mathbf{4})$ were carried out in a 2:1 molar ratio, respectively, in ethanol as solvent and all reactions were monitored by TLC. The most satisfactory results were obtained when the reactions were performed under mild conditions at $78{ }^{\circ} \mathrm{C}$ for 4 hours (Scheme 1). When higher temperatures were used, the formation of a mixture of products not identified by NMR was observed. Also, the increase of the reaction time resultated in a dramatic decline in yields.

After the predicted reaction time, all products were present as solid materials (3c, 3e-g, $4 \mathbf{c}, \mathbf{4 e - f})$, and were filtered from the still hot reaction mixture and the residual solvent was removed under reduced pressure. This simple procedure provided a high degree of purity for the crystallized products. However, for compounds $\mathbf{3 a - b}$, 3d, $\mathbf{3 h}-\mathbf{i}$ and $\mathbf{4 d}$, the solvent was removed under reduced pressure and the solid material had to be recrystallized from methanol, also resulting in compounds with a degree of high purity. Compounds $\mathbf{4 a - b}$ and $\mathbf{4 g - i}$ were not isolated; instead the formation of a complex mixture of insoluble products not identified by NMR, was observed.

In an attempt to develop a methodology that allows the isolation of the product from the reaction of the more reactive nitrogens (hydrazino moiety), we have performed reactions at higher dilutions as well as at lower temperatures (as low as $-70^{\circ} \mathrm{C}$ ). In both cases, we obtained even mixtures of the pyrazolyl-pyridines $\mathbf{3}$ or $\mathbf{4}$ and the pyridine precursor.

Under these reaction conditions, a series of 2-[3alkyl(aryl/heteroaryl)-5-hydroxy-5-trifluoro(chloro) methyl-4,5-dihydro-1H-pyrazol-1-yl]-5-[3-alkyl(aryl/ heteroaryl)-5-hydroxy-5-trifluoro (chloro) methyl-4,5-
dihydro-1H-pyrazol-1-yl-1-arbonyl]pyridines (3a-i and $4 \mathbf{c - f})$ were obtained regiospecifically in one step and in moderate to good yields (62-97\%).

Cristallographic data for compound $\mathbf{3 c}$, reported in this paper has been given to the Cambridge Crystallographic Data Center (CCDC 686704). ${ }^{32}$

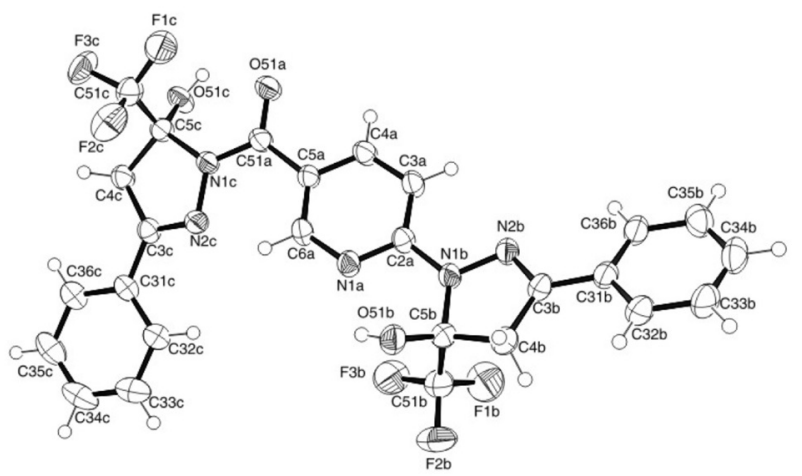

Figure 2. A perspective view of the molecule of the 2-(3-phenyl-5hydroxy-5-trifluoromethyl-4,5-dihydro- $1 H$-pyrazol-1-yl)-5-(3-phenyl5-hydroxy-5-trifluoromethyl-4,5-dihydro- $1 H$-pyrazol-1-yl-1-carbonyl) pyridine (3c) with atoms labeled.

In 2005, we synthesized trifluoro(chloro)methyl substituted heteroaroyl-2-pyrazolines ${ }^{22}$ from the regiospecific reaction of 1,1,1-trifluoro(chloro)-4-methoxy3-alken-2-ones with furoic hydrazide, 2-thiophenecarboxilic hydrazide and isonicotinic acid hydrazide. Subsequently, the dehydration reaction of the resulting 5-trifluoromethyl1-heteroaroyl-3-phenyl-substituted 2-pyrazolines with $\mathrm{P}_{2} \mathrm{O}_{5}$ furnished the corresponding $1 \mathrm{H}$-pyrazoles as a mixture of regioisomers in low yields.

Under reaction conditions similar to those employed for the trifluoromethylated rings, the trichloromethylated analogues ${ }^{30}$ were dehydrated using $\mathrm{P}_{2} \mathrm{O}_{5}$ in chloroform to the respective 5-trichloromethyl substituted aromatic pyrazoles, but also in poor yields (21-29\%) and no mixture of isomers was found in this case.

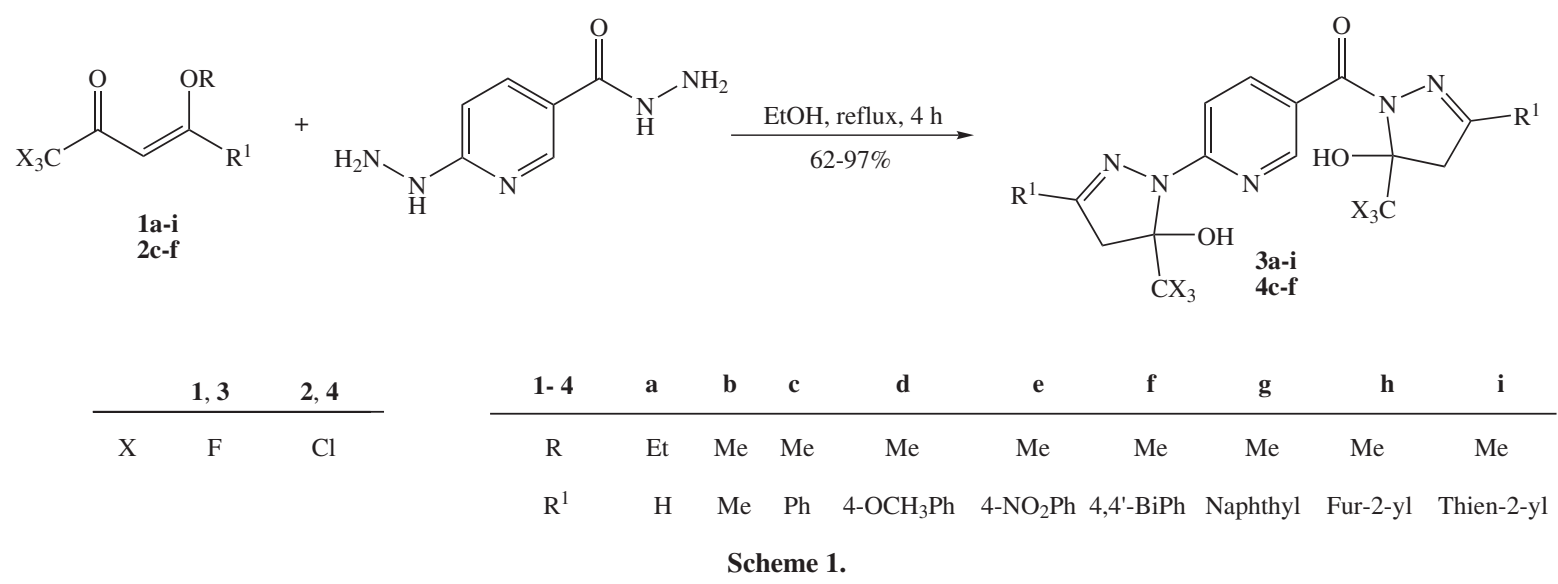




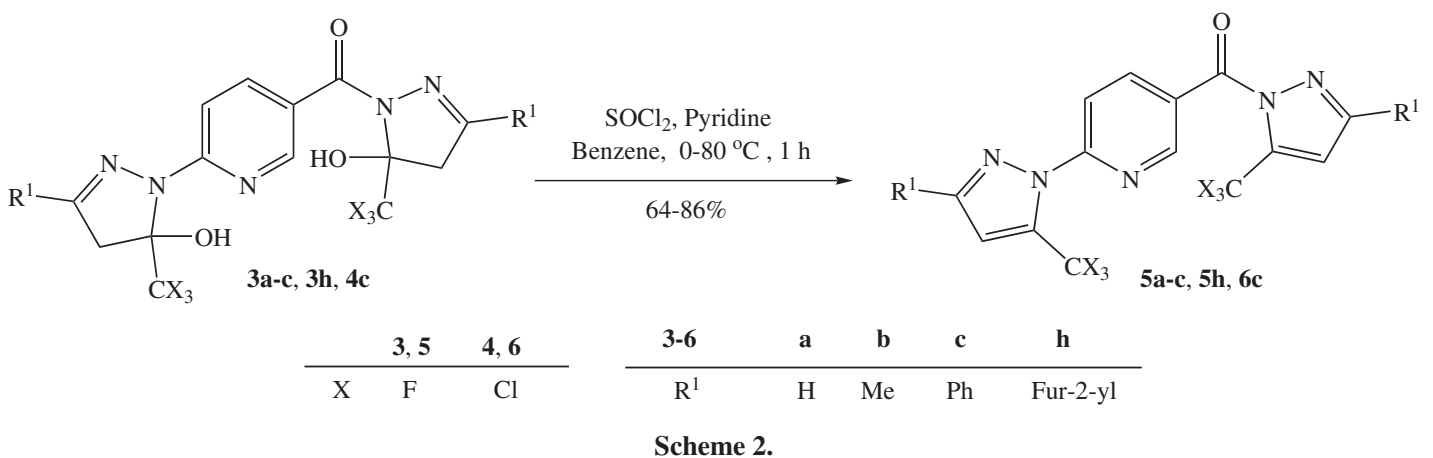

The 1-p-tosyl-2-pyrazolines ${ }^{31}$ were obtained, in 2006, from the cyclocondensation reaction of 3-phenyl- and 4-alkyl(aryl)-1,1,1-trifluoro-4-alkoxy-3-alken-2-ones with $p$-tosylhydrazine in toluene as solvent. Subsequently, the dehydration reaction of 3-(4-fluorophenyl) substituted 2-pyrazoline with thionyl chloride and pyridine in benzene as solvent furnished the corresponding $1 H$-pyrazole.

Thus, we can clearly observe that the trihalomethyl group and the $\mathrm{N}$-substituents promote different chemical behaviors in 5-hydroxy-2-pyrazolines and are decisive factors during water elimination. After a review of the literature and aiming to obtain aromatic pyrazoles containing electron-withdrawing substituents and acid sensitive groups for further biological assays, we chose thionyl chloride as a dehydration agent and reported here the conditions required to accomplish the dehydration of $\mathbf{3 a - c}, \mathbf{3 h}$ and $\mathbf{4} \mathbf{c}$. Due to the relative difficulty in water elimination, because of the simultaneous presence of trihalomethyl and carbonyl group, the pyrazolines, $\mathbf{3 a - c}, \mathbf{3 h}$ and $\mathbf{4 c}$ were dehydrated to give the aromatic pyrazole derivatives $\mathbf{5 a - c}, \mathbf{5 h}$ and $\mathbf{6 c}$ in 64 to $86 \%$ yield only by stirring the mixture of $\mathbf{3 a} \mathbf{a}-\mathbf{c}, \mathbf{3 h}$ and $\mathbf{4 c}$ with thionyl chloride and pyridine at $80{ }^{\circ} \mathrm{C}$ for about $1 \mathrm{~h}$ in benzene as solvent, according to a procedure similar to that described in the literature (Scheme 2). ${ }^{15,30}$

The best reaction conditions, selected physical and spectral data are presented in the experimental part.

The unambiguous ${ }^{1} \mathrm{H}$ and ${ }^{13} \mathrm{C}$ NMR chemical shift assignments of compounds $\mathbf{3 a - i}, \mathbf{4 c - f}, \mathbf{5 a}-\mathbf{c}, \mathbf{5 h}, \mathbf{6 c}$ were performed by comparison with NMR data of other 2-pyrazolines and pyrazoles previously synthesized in our laboratory. ${ }^{28-31}$

Compounds 3a-i show ${ }^{1} \mathrm{H}$ NMR chemical shifts of the diastereotopic methylene protons characteristic of pyrazoline system and as doublets at 3.73 and $3.35 \mathrm{ppm}$, with a geminal coupling constant in the range of ${ }^{2} J 18 \mathrm{~Hz}$; compounds $\mathbf{4 c - f}$ present a pyrazoline system as a doublet at 4.07 and $3.77 \mathrm{ppm}$, with a coupling constant of ${ }^{2} J 18 \mathrm{~Hz}$. The trifluoromethylated heterocycles present the typical ${ }^{13} \mathrm{C}$ chemical shifts of pyrazoline ring carbons at 152.3 and $147.6\left(\mathrm{C}_{3 \mathrm{~B}} / \mathrm{C}_{3 \mathrm{C}}\right), 92.4$ and $91.9\left(\mathrm{C}_{5 \mathrm{~B}} / \mathrm{C}_{5 \mathrm{C}}\right)$ with a constant of ${ }^{2} J 33 \mathrm{~Hz}, 45.1$ and $44.6\left(\mathrm{C}_{4 \mathrm{~B}} / \mathrm{C}_{4 \mathrm{C}}\right), 123.6$ and 123.2
$\left(\mathrm{CF}_{3 \mathrm{~B}} / \mathrm{CF}_{3 \mathrm{C}}\right)$, with ${ }^{1} J 286 \mathrm{~Hz}$ and the trichloromethylated heterocycles of pyrazoline ring carbons at $\delta 152.2$ and $143.0\left(\mathrm{C}_{3 \mathrm{~B}} / \mathrm{C}_{3 \mathrm{C}}\right), 103.0$ and $102.0\left(\mathrm{C}_{5 \mathrm{~B}} / \mathrm{C}_{5 \mathrm{C}}\right), 46.6$ and 46.4 $\left(\mathrm{C}_{4 \mathrm{~B}} / \mathrm{C}_{4 \mathrm{C}}\right), 103.6$ and $103.3\left(\mathrm{CCl}_{3 \mathrm{~B}} / \mathrm{CCl}_{3 \mathrm{C}}\right)$.

Compounds $\mathbf{5 a - c}, \mathbf{5 h}$ were characterized by aromatic protons at 7.47 and $7.26 \mathrm{ppm}$ as a singlet. To aromatic ring carbons at $\delta 152.1$ and $146.8\left(\mathrm{C}_{3 \mathrm{~B}} / \mathrm{C}_{3 \mathrm{C}}\right), 135.8$ and 133.7 $\left(\mathrm{C}_{5 \mathrm{~B}} / \mathrm{C}_{5 \mathrm{C}}\right)$ with a constant ${ }^{2} J 42 \mathrm{~Hz}, 113.3$ and $111.0\left(\mathrm{C}_{4 \mathrm{~B}} /\right.$ $\left.\mathrm{C}_{4 \mathrm{C}}\right), 119.7$ and $119.2\left(\mathrm{CF}_{3 \mathrm{~B}} / \mathrm{CF}_{3 \mathrm{C}}\right)$, with ${ }^{1} J 268 \mathrm{~Hz}$.

Crystallographic data for compound $\mathbf{5 a}$, reported in this paper have been given to the Cambridge Crystallographic Data Center (CCDC 699775). ${ }^{32}$

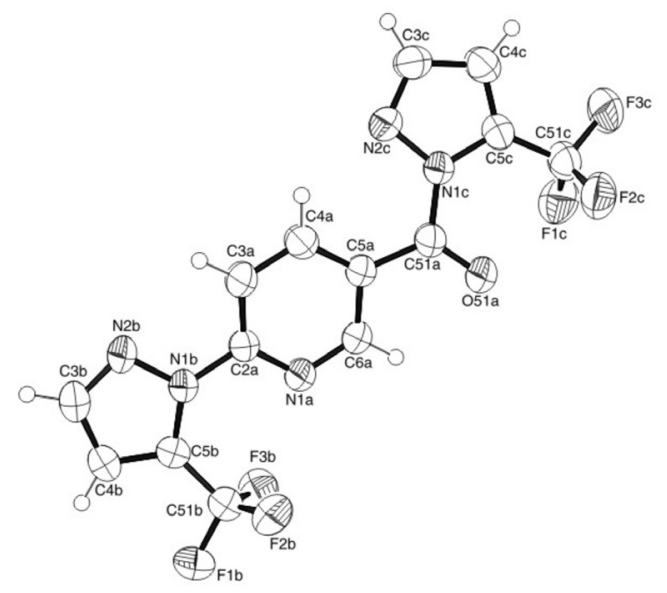

Figure 3. A perspective view of the molecule of 2-(5-trifluoromethyl- $1 H$ pyrazol-1-yl)-5-(5-trifluoromethyl-1 $H$-pyrazol-1-yl-1-carbonyl)pyridine (5a) with atoms labeled.

\section{Conclusions}

In conclusion, we have developed a simple, convenient and one-pot procedure to regiospecifically obtain new 2-[3-alkyl(aryl/heteroaryl)-5-hydroxy-5-trihalomethyl4,5-dihydro-1 $H$-pyrazol-1-yl]-5-[3-alkyl(aryl/heteroaryl)5-hydroxy-5-trihalomethyl-4,5-dihydro- $1 \mathrm{H}$-pyrazol-1yl-1-carbonyl]pyridines ( $\mathbf{3}$ and $\mathbf{4}$ ), as heteropolycycles, without the isolation of intermediates, in good yields and high purity. The intramolecular dehydration furnished, as 
expected, aromatic heterocycles (5a-c, $\mathbf{5} \mathbf{h}$ and $\mathbf{6 c})$, in good yields without the $\mathrm{C}(\mathrm{O})-\mathrm{N}$ bond cleavage.

\section{Acknowledgments}

The authors thank for the financial support from Conselho Nacional de Desenvolvimento Científico e Tecnológico (CNPq) and for fellowship (T.S.M.) from the Coordenação de Aperfeiçoamento de Pessoal de Nível Superior (CAPES); FATECIENS are also acknowledged.

\section{Experimental}

Unless otherwise indicated, all common reagents and solvents were used as obtained from commercial suppliers without further purification. The melting points were determined on a Reichert Thermovar apparatus and are uncorrected. ${ }^{1} \mathrm{H}$ and ${ }^{13} \mathrm{C}$ NMR spectra were acquired on a Bruker DPX $200\left({ }^{1} \mathrm{H}\right.$ at $200.13 \mathrm{MHz})$ and Bruker DPX $400\left({ }^{1} \mathrm{H}\right.$ at $400.13 \mathrm{MHz}$ and ${ }^{13} \mathrm{C}$ at $100.61 \mathrm{MHz}$ ) spectrometer, $5 \mathrm{~mm}$ sample tubes, $298 \mathrm{~K}$, digital resolution $\pm 0.01 \mathrm{ppm}$, in $\mathrm{CDCl}_{3}$ or DMSO- $d_{6}$ and using TMS as internal reference. The CHN elemental analyses were performed on a Perkin-Elmer $2400 \mathrm{CHN}$ elemental analyzer (São Paulo University, USP/Brazil).

General procedure for the synthesis of 2-[3-alkyl(aryl/ heteroaryl)-5-hydroxy-5-trihalomethyl-4,5-dihydro- $1 \mathrm{H}$ pyrazol-1-yl]-5-[3-alkyl(aryl/heteroaryl)-5-hydroxy-5trihalomethyl-4,5-dihydro-1H-pyrazol-1-yl-1carbonyl]pyridines ( $\mathbf{3} \boldsymbol{a}-\boldsymbol{i}$ and $\mathbf{4 c}-\mathrm{f}$ )

A solution of 1,1,1-trihalo-4-alkyl (aryl/heteroaryl)-4methoxy-3-alken-2-one (5 mmol) and 6-hydrazinonicotinic hydrazide hydrate $(2.5 \mathrm{mmol})$ in $15 \mathrm{~mL}$ of ethanol was refluxed for $4 \mathrm{~h}$ under stirring. The products $\mathbf{3 c}, \mathbf{3 e -}-\mathbf{g}, \mathbf{4 c}$, 4e-f were obtained as solids and filtered while still hot and residual solvent was removed under reduced pressure. All compounds presented a high degree of purity and did not undergo recrystallization. For compounds 3a-b, 3d, 3h-i, $\mathbf{4 d}$ the solvent was evaporated, furnishing solids that were recrystallized from methanol.

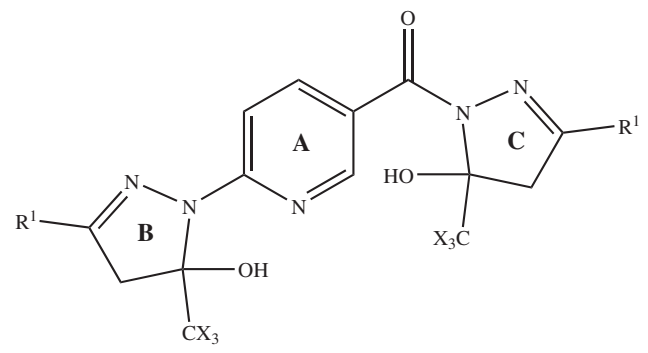

2-(5-Hydroxy-5-trifluoromethyl-4,5-dihydro-1H-pyrazol1-yl)-5-(5-hydroxy-5-trifluoromethyl-4,5-dihydro-1Hpyrazol-1-yl-1-carbonyl)pyridine (3a)

Yellow solid; yield 68\%; mp 165-167 ${ }^{\circ} \mathrm{C} .{ }^{1} \mathrm{H}$ NMR $\left(\mathrm{CDCl}_{3}\right): \delta 8.75\left(\mathrm{~s}, 1 \mathrm{H}, 6_{\mathrm{A}}\right) ; 8.22-8.16\left(\mathrm{~m}, 1 \mathrm{H}, 4_{\mathrm{A}}\right) ; 7.40-$ $7.36\left(\mathrm{~m}, 1 \mathrm{H}, 3_{\mathrm{A}}\right) ; 7.02\left(\mathrm{~s}, 2 \mathrm{H}, 3_{\mathrm{B}}\right.$ and $\left.3_{\mathrm{C}}\right) ; 3.45\left(\mathrm{~d}, 1 \mathrm{H}, 4_{\mathrm{B}} / 4_{\mathrm{C}}\right.$, $J 18.3) ; 3.38\left(\mathrm{~d}, 1 \mathrm{H}, 4_{\mathrm{B}} / 4_{\mathrm{C}}, J 19.8\right) ; 3.25\left(\mathrm{~d}, 1 \mathrm{H}, 4_{\mathrm{B}}, 4_{\mathrm{C}}, J\right.$ 18.3); $3.20\left(\mathrm{~d}, 1 \mathrm{H}, 4_{\mathrm{B}}, / 4_{\mathrm{C}}, J 19.8\right) .{ }^{13} \mathrm{C}$ NMR (DMSO- $\left.d_{6}\right)$ : $\delta 164.9(\mathrm{CO}) ; 156.3\left(\mathrm{C}_{2 \mathrm{~A}}\right) ; 148.5\left(\mathrm{C}_{3 \mathrm{~B}} / \mathrm{C}_{3 \mathrm{C}}\right) ; 144.4\left(\mathrm{C}_{6 \mathrm{~A}}\right)$; $139.5\left(\mathrm{C}_{3 \mathrm{~B}} / \mathrm{C}_{3 \mathrm{C}}\right) ; 123.7\left(\mathrm{q},{ }^{1} J_{\mathrm{C}-\mathrm{F}} 286.8, \mathrm{CF}_{3-\mathrm{B}} / \mathrm{CF}_{3-\mathrm{C}}\right) ; 123.3$ $\left(\mathrm{q},{ }^{1} \mathrm{~J}_{\mathrm{C}-\mathrm{F}} 285.4 \mathrm{CF}_{3-\mathrm{B}} / \mathrm{CF}_{3-\mathrm{C}}\right) ; 122.8\left(\mathrm{C}_{5 \mathrm{~A}}\right) ; 109.2\left(\mathrm{C}_{3 \mathrm{~A}}\right) ; 90.6$ $\left(\mathrm{q},{ }^{2} J_{\mathrm{C}-\mathrm{F}} 32.5, \mathrm{C}_{5 \mathrm{~B}} / \mathrm{C}_{5 \mathrm{C}}\right) ; 90.0\left(\mathrm{q},{ }^{2} J_{\mathrm{C}-\mathrm{F}} 33.6, \mathrm{C}_{5 \mathrm{~B}} / \mathrm{C}_{5 \mathrm{C}}\right) ; 46.1$ $\left(\mathrm{C}_{4 \mathrm{~B}} / \mathrm{C}_{4 \mathrm{C}}\right) ; 45.7\left(\mathrm{C}_{4 \mathrm{~B}} / \mathrm{C}_{4 \mathrm{C}}\right)$. Anal. Calc. for $\mathrm{C}_{14} \mathrm{H}_{11} \mathrm{~F}_{6} \mathrm{~N}_{5} \mathrm{O}_{3}$ (411.08): C, 40.89.; H, 2.70; N, 17.03\%. Found: C, 41.03; $\mathrm{H}, 2.64 ; \mathrm{N}, 17.05 \%$.

2-(3-Methyl-5-hydroxy-5-trifluoromethyl-4,5-dihydro- $1 \mathrm{H}$ pyrazol-1-yl)-5-(3-methyl-5-hydroxy-5-trifluoromethyl-4,5dihydro-1H-pyrazol-1-yl-1-carbonyl)pyridine (3b)

White solid; yield 80\%; mp 106-108 ${ }^{\circ} \mathrm{C} .{ }^{1} \mathrm{H}$ NMR $\left(\mathrm{CDCl}_{3}\right): \delta 8.75-8.73\left(\mathrm{~m} .1 \mathrm{H}, 6_{\mathrm{A}}\right) ; 8.20-8.13\left(\mathrm{~m}, 1 \mathrm{H}, 4_{\mathrm{A}}\right)$; $7.31\left(\mathrm{~d}, J 9,1 \mathrm{H}, 3_{\mathrm{A}}\right) ; 3.34\left(\mathrm{~d}, J 18.8,1 \mathrm{H}, 4_{\mathrm{B}} / 4_{\mathrm{C}}\right) ; 3.30(\mathrm{~d}, J$ $\left.19,1 \mathrm{H}, 4_{\mathrm{B}} / 4_{\mathrm{C}}\right) ; 3.17\left(\mathrm{~d}, J 18.8,1 \mathrm{H}, 4_{\mathrm{B}} / 4_{\mathrm{C}}\right) ; 3.11(\mathrm{~d}, J 19$, $\left.1 \mathrm{H}, 4_{\mathrm{B}}, / 4_{\mathrm{C}}\right) ; 2.10\left(\mathrm{~s}, 3 \mathrm{H}, \mathrm{CH}_{3-\mathrm{B}} / \mathrm{CH}_{3-\mathrm{C}}\right) ; 2.08\left(\mathrm{~s}, 3 \mathrm{H}, \mathrm{CH}_{3-\mathrm{B}} /\right.$ $\left.\mathrm{CH}_{3-\mathrm{C}}\right) \cdot{ }^{13} \mathrm{CNMR}\left(\mathrm{CDCl}_{3}\right): \delta 168.3(\mathrm{CO}) ; 157.8\left(\mathrm{C}_{2 \mathrm{~A}}\right) ; 155.0$ $\left(\mathrm{C}_{3 \mathrm{~B}} / \mathrm{C}_{3 \mathrm{C}}\right) ; 152.9\left(\mathrm{C}_{6 \mathrm{~A}}\right) ; 149.2\left(\mathrm{C}_{3 \mathrm{~B}} / \mathrm{C}_{3 \mathrm{C}}\right) ; 140.1\left(\mathrm{C}_{4 \mathrm{~A}}\right) ; 123.6$ $\left(\mathrm{q},{ }^{1} J_{\mathrm{C}-\mathrm{F}} 287.9, \mathrm{CF}_{3-\mathrm{B}} / \mathrm{CF}_{3-\mathrm{C}}\right) ; 123.2\left(\mathrm{q},{ }^{1} J_{\mathrm{C}-\mathrm{F}} 286.6, \mathrm{CF}_{3-\mathrm{B}} /\right.$ $\left.\mathrm{CF}_{3-\mathrm{C}}\right) ; 120.4\left(\mathrm{C}_{5 \mathrm{~A}}\right) ; 109.5\left(\mathrm{C}_{3 \mathrm{~A}}\right) ; 92.6\left(\mathrm{q},{ }^{2} J_{\mathrm{C}-\mathrm{F}} 33.6, \mathrm{C}_{5 \mathrm{~B}} /\right.$ $\left.\mathrm{C}_{5 \mathrm{C}}\right) ; 92.5\left(\mathrm{q},{ }^{2} J_{\mathrm{C}-\mathrm{F}} 34, \mathrm{C}_{5 \mathrm{~B}} / \mathrm{C}_{5 \mathrm{C}}\right) ; 47.5\left(\mathrm{C}_{4 \mathrm{~B}} / \mathrm{C}_{4 \mathrm{C}}\right) ; 46.5\left(\mathrm{C}_{4 \mathrm{~B}} /\right.$ $\left.\mathrm{C}_{4 \mathrm{C}}\right)$; $15.6\left(\mathrm{CH}_{3-\mathrm{B}}\right.$ andCH $\left.\mathrm{CH}_{3-\mathrm{C}}\right)$. Anal. Calc. for $\mathrm{C}_{16} \mathrm{H}_{15} \mathrm{~F}_{6} \mathrm{~N}_{5} \mathrm{O}_{3}$ (439.11): C, 43.74.; H, 3.44; N, 15.94\%. Found: C, 43.72; $\mathrm{H}, 3.24 ; \mathrm{N}, 16.19 \%$.

2-(3-Phenyl-5-hydroxy-5-trifluoromethyl-4,5-dihydro-1Hpyrazol-1-yl)-5-(3-phenyl-5-hydroxy-5-trifluoromethyl-4,5dihydro-1H-pyrazol-1-yl-1-carbonyl)pyridine (3c)

Yellow solid; yield 97\%; mp 225-227 ${ }^{\circ} \mathrm{C} .{ }^{1} \mathrm{H}$ NMR $\left(\mathrm{CDCl}_{3}\right): \delta 8.91-8.88\left(\mathrm{~m}, 1 \mathrm{H}, 6_{\mathrm{A}}\right) ; 8.37-8.31\left(\mathrm{~m}, 1 \mathrm{H}, 4_{\mathrm{A}}\right)$; 7.78-7.67 (m, $4 \mathrm{H}, \mathrm{Ph}) ; 7.56\left(\mathrm{~d}, J 9,1 \mathrm{H}, 3_{\mathrm{A}}\right) ; 7.48-7.44(\mathrm{~m}, 6 \mathrm{H}$, $\mathrm{Ph}) ; 3.80\left(\mathrm{~d}, J 18.5,1 \mathrm{H}, 4_{\mathrm{B}} / 4_{\mathrm{C}}\right) ; 3.75\left(\mathrm{~d}, J 19.5,1 \mathrm{H}, 4_{\mathrm{B}} / 4_{\mathrm{C}}\right)$; $3.64\left(\mathrm{~d}, J 18.5,1 \mathrm{H}, 4_{\mathrm{B}}, / 4_{\mathrm{C}}\right) ; 3.56\left(\mathrm{~d}, J 19.5,1 \mathrm{H} .4_{\mathrm{B}}, 4_{\mathrm{C}}\right) \cdot{ }^{13} \mathrm{C}$ NMR (DMSO- $\left.d_{6}\right): \delta 164.5(\mathrm{CO}) ; 156.0\left(\mathrm{C}_{2 \mathrm{~A}}\right) ; 152.3\left(\mathrm{C}_{3 \mathrm{~B}} /\right.$ $\left.\mathrm{C}_{3 \mathrm{C}}\right) ; 150.9\left(\mathrm{C}_{6 \mathrm{~A}}\right) ; 148.9\left(\mathrm{C}_{3 \mathrm{~B}} / \mathrm{C}_{3 \mathrm{C}}\right) ; 139.8\left(\mathrm{C}_{4 \mathrm{~A}}\right) ; 130.7(\mathrm{Ph})$; $130.4(\mathrm{Ph}) ; 130.2(\mathrm{Ph}) ; 130.0(\mathrm{Ph}) ; 128.8(\mathrm{Ph}) ; 128.7(\mathrm{Ph})$; $126.7(\mathrm{Ph}) ; 126.3(\mathrm{Ph}) ; 123.7\left(\mathrm{q},{ }^{1} J_{\mathrm{C}-\mathrm{F}} 286.7, \mathrm{CF}_{3-\mathrm{B}} / \mathrm{CF}_{3-\mathrm{C}}\right)$; $123.3\left(\mathrm{q},{ }^{1} J_{\mathrm{C}-\mathrm{F}} 285.2, \mathrm{CF}_{3-\mathrm{B}} / \mathrm{CF}_{3-\mathrm{C}}\right) ; 122.8\left(\mathrm{C}_{5 \mathrm{~A}}\right) ; 109.2\left(\mathrm{C}_{3 \mathrm{~A}}\right)$; $92.7\left(\mathrm{q},{ }^{2} J_{\mathrm{C}-\mathrm{F}} 32.9, \mathrm{C}_{5 \mathrm{~B}} / \mathrm{C}_{5 \mathrm{C}}\right.$ ); $92.2\left(\mathrm{q},{ }^{2} J_{\mathrm{C}-\mathrm{F}} 33.7, \mathrm{C}_{5 \mathrm{~B}} / \mathrm{C}_{5 \mathrm{C}}\right.$ ); $44.4\left(\mathrm{C}_{4 \mathrm{~B}} / \mathrm{C}_{4 \mathrm{C}}\right) ; 44.0\left(\mathrm{C}_{4 \mathrm{~B}} / \mathrm{C}_{4 \mathrm{C}}\right)$. Anal. Calc. for $\mathrm{C}_{26} \mathrm{H}_{19} \mathrm{~F}_{6} \mathrm{~N}_{5} \mathrm{O}_{3}$ (563.14): C, 55.42; H, 3.40; N, 12.43\%. Found: C, 55.48; H, $3.52 ; \mathrm{N}, 12.41 \%$.

Figure 4. 
2-[3-(4-Methoxyphenyl)-5-hydroxy-5-trifluoromethyl4,5-dihydro-1H-pyrazol-1-yl]-5-[3-(4-methoxyphenyl)-5hydroxy-5-trifluoromethyl-4,5-dihydro-1H-pyrazol-1-yl1-carbonyl]pyridine $(\mathbf{3 d})$

Yellow solid; yield 80\%; mp 155-157 ${ }^{\circ} \mathrm{C} .{ }^{1} \mathrm{H}$ NMR $\left(\mathrm{CDCl}_{3}\right): \delta 8.91-8.87\left(\mathrm{~m}, 1 \mathrm{H}, 6_{\mathrm{A}}\right) ; 8.36-8.29\left(\mathrm{~m}, 1 \mathrm{H}, 4_{\mathrm{A}}\right)$; 7.71-7.60 (m, 4H, Ph); 7.52 (d, J 9, 1H, $3_{\mathrm{A}}$ ); 6.98-6.93 $(\mathrm{m}, 4 \mathrm{H}, \mathrm{Ph}) ; 3.87\left(\mathrm{~s}, 3 \mathrm{H}, \mathrm{OCH}_{3-\mathrm{B}} / \mathrm{OCH}_{3-\mathrm{C}}\right) ; 3.86(\mathrm{~s}, 3 \mathrm{H}$, $\left.\mathrm{OCH}_{3-\mathrm{B}} / \mathrm{OCH}_{3-\mathrm{C}}\right) ; 3.76\left(\mathrm{~d}, J 18.5,1 \mathrm{H}, 4_{\mathrm{B}} / 4_{\mathrm{C}}\right) ; 3.71(\mathrm{~d}, J$ $\left.18.3,1 \mathrm{H}, 4_{\mathrm{B}} / 4_{\mathrm{C}}\right) ; 3.60\left(\mathrm{~d}, J 18.5,1 \mathrm{H}, 4_{\mathrm{B}} / 4_{\mathrm{C}}\right) ; 3.52(\mathrm{~d}, J$ $\left.18.3,1 \mathrm{H}, 4_{\mathrm{B}}, / 4_{\mathrm{C}}\right) .{ }^{13} \mathrm{C} \mathrm{NMR}\left(\mathrm{CDCl}_{3}\right): \delta 168.2(\mathrm{CO}) ; 162.0$ $\left(\mathrm{C}_{2 \mathrm{~A}}\right) ; 161.4\left(\mathrm{C}_{3 \mathrm{~B}} / \mathrm{C}_{3 \mathrm{C}}\right) ; 157.7\left(\mathrm{C}_{6 \mathrm{~A}}\right) ; 153.3\left(\mathrm{C}_{3 \mathrm{~B}} / \mathrm{C}_{3 \mathrm{C}}\right) ; 151.4$ $\left(\mathrm{C}_{4 \mathrm{~A}}\right) 149.5(\mathrm{Ph}) ; 140.4(\mathrm{Ph}) ; 128.4(\mathrm{Ph}) ; 127.9(\mathrm{Ph}) ; 123.7$ $\left(\mathrm{q},{ }^{1} J_{\mathrm{C}-\mathrm{F}} 295.2, \mathrm{CF}_{3-\mathrm{B}} / \mathrm{CF}_{3-\mathrm{C}}\right) ; 123.4\left(\mathrm{q},{ }^{1} J_{\mathrm{C}-\mathrm{F}} 287.5, \mathrm{CF}_{3-\mathrm{B}} /\right.$ $\left.\mathrm{CF}_{3-\mathrm{C}}\right) ; 123.1(\mathrm{Ph}) ; 122.2(\mathrm{Ph}) ; 120.6\left(\mathrm{C}_{5 \mathrm{~A}}\right) ; 114.3(\mathrm{Ph})$; $114.2(\mathrm{Ph}) ; 109.5\left(\mathrm{C}_{3 \mathrm{~A}}\right) ; 93.1\left(\mathrm{q},{ }^{2} J_{\mathrm{C}-\mathrm{F}} 33.2, \mathrm{C}_{5 \mathrm{~B}} / \mathrm{C}_{5 \mathrm{C}}\right) ; 92.9$ $\left(\mathrm{q},{ }^{2} J_{\mathrm{C}-\mathrm{F}} 33.9, \mathrm{C}_{5 \mathrm{~B}} / \mathrm{C}_{5 \mathrm{C}}\right) ; 55.3\left(\mathrm{OCH}_{3-\mathrm{B}}\right.$ and $\left.\mathrm{OCH}_{3-\mathrm{C}}\right) ; 43.9\left(\mathrm{C}_{4 \mathrm{~B}} /\right.$ $\left.\mathrm{C}_{4 \mathrm{C}}\right) ; 43.0\left(\mathrm{C}_{4 \mathrm{~B}} / \mathrm{C}_{4 \mathrm{C}}\right)$. Anal. Calc. for $\mathrm{C}_{28} \mathrm{H}_{23} \mathrm{~F}_{6} \mathrm{~N}_{5} \mathrm{O}_{5}(623.16)$ : C, 53.94; H, 3.72; N, 11.23\%. Found: C, 53.95; H, 3.71; $\mathrm{N}, 11.25 \%$.

2-[3-(4-Nitrophenyl)-5-hydroxy-5-trifluoromethyl-4,5dihydro-1H-pyrazol-1-yl]-5-[3-(4-nitrophenyl)-5-hydroxy5-trifluoromethyl-4,5-dihydro-1H-pyrazol-1-yl-1-carbonyl] pyridine $(3 \boldsymbol{e})$

Yellow solid; yield 70\%; mp 230-232 ${ }^{\circ} \mathrm{C} .{ }^{1} \mathrm{H}$ NMR $\left(\mathrm{CDCl}_{3}\right): \delta 8.90-8.87\left(\mathrm{~m}, 1 \mathrm{H}, 6_{\mathrm{A}}\right) ; 8.37-8.29(\mathrm{~m}, 5 \mathrm{H}, \mathrm{Ph}$ and $\left.4_{\mathrm{A}}\right) ; 7.93-7.82(\mathrm{~m}, 4 \mathrm{H}, \mathrm{Ph}) ; 7.61\left(\mathrm{~d}, J 9,1 \mathrm{H}, 3_{\mathrm{A}}\right) ; 3.84$ $\left(\mathrm{d}, J 18.5,1 \mathrm{H}, 4_{\mathrm{B}} / 4_{\mathrm{C}}\right) ; 3.80\left(\mathrm{~d}, J 18.8,1 \mathrm{H}, 4_{\mathrm{B}} / 4_{\mathrm{C}}\right) ; 3.67(\mathrm{~d}$, $\left.J 18.5,1 \mathrm{H}, 4_{\mathrm{B}}, 4_{\mathrm{C}}\right) ; 3.60\left(\mathrm{~d}, J 18.3,1 \mathrm{H}, 4_{\mathrm{B}} / 4_{\mathrm{C}}\right) \cdot{ }^{13} \mathrm{C} \mathrm{NMR}$ $\left(\right.$ DMSO- $\left._{6}\right): \delta 164.6(\mathrm{CO}) ; 155.7\left(\mathrm{C}_{2 \mathrm{~A}}\right) ; 150.8\left(\mathrm{C}_{3 \mathrm{~B}} / \mathrm{C}_{3 \mathrm{C}}\right)$; $149.0\left(\mathrm{C}_{6 \mathrm{~A}}\right) ; 148.2\left(\mathrm{C}_{3 \mathrm{~B}} / \mathrm{C}_{3 \mathrm{C}}\right) ; 147.8\left(\mathrm{C}_{4 \mathrm{~A}}\right) ; 139.8(\mathrm{Ph})$; $136.5(\mathrm{Ph}) ; 136.0(\mathrm{Ph}) ; 127.8(\mathrm{Ph}) ; 127.3(\mathrm{Ph}) ; 123.9$ $(\mathrm{Ph}) ; 123.4(\mathrm{Ph}) ; 123.4\left(\mathrm{q},{ }^{1} J_{\mathrm{C}-\mathrm{F}} 286.8, \mathrm{CF}_{3-\mathrm{B}} / \mathrm{CF}_{3-\mathrm{C}}\right) ; 123.2$ $\left(\mathrm{C}_{5 \mathrm{~A}}\right) ; 123.1\left(\mathrm{q},{ }^{1} \mathrm{~J}_{\mathrm{C}-\mathrm{F}} 285.4, \mathrm{CF}_{3-\mathrm{B}} / \mathrm{CF}_{3-\mathrm{C}}\right) ; 109.5\left(\mathrm{C}_{3 \mathrm{~A}}\right) ; 93.3$ $\left(\mathrm{q},{ }^{2} J_{\mathrm{C}-\mathrm{F}} 32.9, \mathrm{C}_{5 \mathrm{~B}} / \mathrm{C}_{5 \mathrm{C}}\right) ; 92.7\left(\mathrm{q},{ }^{2} J_{\mathrm{C}-\mathrm{F}} 33.6, \mathrm{C}_{5 \mathrm{~B}} / \mathrm{C}_{5 \mathrm{C}}\right) ; 44.1$ $\left(\mathrm{C}_{4 \mathrm{~B}} / \mathrm{C}_{4 \mathrm{C}}\right) ; 43.8\left(\mathrm{C}_{4 \mathrm{~B}} / \mathrm{C}_{4 \mathrm{C}}\right)$. Anal. Calc. for $\mathrm{C}_{26} \mathrm{H}_{17} \mathrm{~F}_{6} \mathrm{~N}_{7} \mathrm{O}_{7}$ (653.11): C, 47.79; H, 2.62; N, 15.00\%. Found: C, 48.18; $\mathrm{H}, 2.47 ; \mathrm{N}, 15.40 \%$.

2-[3-(4,4'-Biphenyl)-5-hydroxy-5-trifluoromethyl-4,5dihydro-1H-pyrazol-1-yl]-5-[3-(4,4'-biphenyl)-5-hydroxy5-trifluoromethyl-4,5-dihydro-1H-pyrazol-1-yl-1-carbonyl] pyridine $(\mathbf{3} f)$

Yellow solid; yield 85\%; mp 193-195 ${ }^{\circ} \mathrm{C} .{ }^{1} \mathrm{H}$ NMR $\left(\mathrm{CDCl}_{3}\right): \delta 8.94-8.92\left(\mathrm{~m}, 1 \mathrm{H}, 6_{\mathrm{A}}\right) ; 8.39-8.35\left(\mathrm{~m}, 1 \mathrm{H}, 4_{\mathrm{A}}\right)$; 8.07-8.03 (m, 1H, $\left.3_{\mathrm{A}}\right) ; 7.85-7.39$ (m, 18H, BiPh); 3.83 (d, $J$ $\left.18,1 \mathrm{H}, 4_{\mathrm{B}} / 4_{\mathrm{C}}\right) ; 3.80\left(\mathrm{~d}, J 18.5,1 \mathrm{H}, 4_{\mathrm{B}} / 4_{\mathrm{C}}\right) ; 3.67(\mathrm{~d}, J 18,1 \mathrm{H}$, $\left.4_{\mathrm{B}} / 4_{\mathrm{C}}\right) ; 3.64\left(\mathrm{~d}, J 18.5,1 \mathrm{H}, 4_{\mathrm{B}} / 4_{\mathrm{C}}\right) \cdot{ }^{13} \mathrm{C}$ NMR (DMSO- $\left.d_{6}\right): \delta$ $164.3(\mathrm{CO}) ; 156.2\left(\mathrm{C}_{2 \mathrm{~A}}\right) ; 156.0(\mathrm{BiPh}) ; 152.0\left(\mathrm{C}_{3 \mathrm{~B}} / \mathrm{C}_{3 \mathrm{C}}\right) ; 150.6$ $(\mathrm{BiPh}) ; 150.2\left(\mathrm{C}_{6 \mathrm{~A}}\right) ; 148.9\left(\mathrm{C}_{3 \mathrm{~B}} / \mathrm{C}_{3 \mathrm{C}}\right) ; 146.2(\mathrm{BiPh}) ; 142.1$
(BiPh); $141.6(\mathrm{BiPh}) ; 141.5(\mathrm{BiPh}) ; 139.1(\mathrm{BiPh}) ; 139.0$ $(\mathrm{BiPh}) ; 137.0(\mathrm{BiPh}) ; 129.5(\mathrm{BiPh}) ; 128.9(\mathrm{BiPh}) ; 127.9$ (BiPh); 127.3 (BiPh); $126.9(\mathrm{BiPh}) ; 126.9(\mathrm{BiPh}) ; 126.6$ $(\mathrm{BiPh}) ; 123.7\left(\mathrm{q},{ }^{1} J_{\mathrm{C}-\mathrm{F}} 286.1, \mathrm{CF}_{3-\mathrm{B}} / \mathrm{CF}_{3-\mathrm{C}}\right) ; 123.3\left(\mathrm{q},{ }^{1} J_{\mathrm{C}-\mathrm{F}} 286.8\right.$, $\left.\mathrm{CF}_{3-\mathrm{B}} / \mathrm{CF}_{3-\mathrm{C}}\right) ; 121.5\left(\mathrm{C}_{5 \mathrm{~A}}\right) ; 110.0\left(\mathrm{C}_{3 \mathrm{~A}}\right) ; 92.8\left(\mathrm{q},{ }^{2} J_{\mathrm{C}-\mathrm{F}} 32.9, \mathrm{C}_{5 \mathrm{~B}} /\right.$ $\left.\mathrm{C}_{5 \mathrm{C}}\right) ; 92.7\left(\mathrm{q},{ }^{2} \mathrm{~J}_{\mathrm{C}-\mathrm{F}} 32.9, \mathrm{C}_{5 \mathrm{~B}} / \mathrm{C}_{5 \mathrm{C}}\right) ; 44.4\left(\mathrm{C}_{4 \mathrm{~B}} / \mathrm{C}_{4 \mathrm{C}}\right) ; 44.0\left(\mathrm{C}_{4 \mathrm{~B}} /\right.$ $\mathrm{C}_{4 \mathrm{C}}$ ). Anal. Calc. for $\mathrm{C}_{38} \mathrm{H}_{27} \mathrm{~F}_{6} \mathrm{~N}_{5} \mathrm{O}_{5}(715.20): \mathrm{C}, 63.28 ; \mathrm{H}, 3.80$; N, 9.79\%. Found: C, $63.40 ; \mathrm{H}, 3.80$; N, 9.41\%.

2-[3-(1-Naphthyl)-5-hydroxy-5-trifluoromethyl-4,5dihydro-1H-pyrazol-1-yl]-5-[3-(1-naphthyl)-5-hydroxy-5trifluoromethyl-4,5-dihydro-1H-pyrazol-1-yl-1-carbonyl] pyridine (3g)

Yellow solid; yield 67\%; mp 137-139 ${ }^{\circ} \mathrm{C} .{ }^{1} \mathrm{H}$ NMR $\left(\mathrm{CDCl}_{3}\right): \delta 9.31-9.27\left(\mathrm{~m}, 1 \mathrm{H}, 6_{\mathrm{A}}\right) ; 8.82-8.80\left(\mathrm{~m}, 1 \mathrm{H}, 4_{\mathrm{A}}\right)$; 8.32-8.27 (m, 2H, naphthyl); 8.20-8.19 (m, $\left.1 \mathrm{H}, 3_{\mathrm{A}}\right) ; 8.10-$ 8.00 ( $\mathrm{m}, 4 \mathrm{H}$, naphthyl); 7.95-7.89 (m, 2H, naphthyl); 7.66-7.52 (m, 6H, naphthyl); $4.26\left(\mathrm{~d}, J 19.2,1 \mathrm{H}, 4_{\mathrm{B}} / 4_{\mathrm{C}}\right.$ ); $4.22\left(\mathrm{~d}, J 18.9,1 \mathrm{H}, 4_{\mathrm{B}} / 4_{\mathrm{C}}\right) ; 3.94\left(\mathrm{~d}, J 19.2,1 \mathrm{H}, 4_{\mathrm{B}} / 4_{\mathrm{C}^{\mathrm{C}}}\right)$; $3.85\left(\mathrm{~d}, J 18.9,1 \mathrm{H}, 4_{\mathrm{B}} / 4_{\mathrm{C}}\right) \cdot{ }^{13} \mathrm{C}$ NMR (DMSO- $\left.d_{6}\right): \delta 165.1$ $(\mathrm{CO}) ; 156.0\left(\mathrm{C}_{2 \mathrm{~A}}\right) ; 152.9\left(\mathrm{C}_{3 \mathrm{~B}} / \mathrm{C}_{3 \mathrm{C}}\right) ; 151.6\left(\mathrm{C}_{6 \mathrm{~A}}\right) ; 148.6$ $\left(\mathrm{C}_{3 \mathrm{~B}} / \mathrm{C}_{3 \mathrm{C}}\right) ; 139.6\left(\mathrm{C}_{4 \mathrm{~A}}\right) ; 133.6$ (naphthyl); 131.5 (naphthyl); 130.9 (naphthyl); 129.8 (naphthyl); 129.6 (naphthyl); 128.8 (naphthyl); 127.8 (naphthyl); 127.4 (naphthyl); 126.2 (naphthyl); 125.9 (naphthyl); 125.2 (naphthyl); $123.8\left(\mathrm{q},{ }^{1} J_{\mathrm{C}-\mathrm{F}} 287.6, \mathrm{CF}_{3-\mathrm{B}} / \mathrm{CF}_{3-\mathrm{C}}\right) ; 123.6\left(\mathrm{q},{ }^{1} J_{\mathrm{C}-\mathrm{F}} 286.1\right.$, $\left.\mathrm{CF}_{3-\mathrm{B}} / \mathrm{CF}_{3-\mathrm{C}}\right) ; 123.4$ (naphthyl); $123.2\left(\mathrm{C}_{5 \mathrm{~A}}\right) ; 109.3\left(\mathrm{C}_{3 \mathrm{~A}}\right)$; $91.6\left(\mathrm{q},{ }^{2} J_{\mathrm{C}-\mathrm{F}} 32.9, \mathrm{C}_{5 \mathrm{~B}} / \mathrm{C}_{5 \mathrm{C}}\right) ; 90.9\left(\mathrm{q},{ }^{2} J_{\mathrm{C}-\mathrm{F}} 34, \mathrm{C}_{5 \mathrm{~B}} / \mathrm{C}_{5 \mathrm{C}}\right) ; 46.8$ $\left(\mathrm{C}_{4 \mathrm{~B}} / \mathrm{C}_{4 \mathrm{C}}\right) ; 46.4\left(\mathrm{C}_{4 \mathrm{~B}} / \mathrm{C}_{4 \mathrm{C}}\right)$. Anal. Calc. for $\mathrm{C}_{34} \mathrm{H}_{23} \mathrm{~F}_{6} \mathrm{~N}_{5} \mathrm{O}_{3}$ (663.17): C, 61.54; H, 3.49; N, 10.55\%. Found: C, 61.94; $\mathrm{H}, 3.89 ; \mathrm{N}, 10.18 \%$.

2-[3-(Fur-2-yl)-5-hydroxy-5-trifluoromethyl-4,5dihydro-1H-pyrazol-1-yl]-5-[3-(fur-2-yl)-5-hydroxy-5trifluoromethyl-4,5-dihydro-1H-pyrazol-1-yl-1-carbonyl] pyridine (3h)

Brown solid; yield 78\%; mp 196-198 ${ }^{\circ} \mathrm{C} .{ }^{1} \mathrm{H}$ NMR $\left(\mathrm{CDCl}_{3}\right): \delta 8.88-8.84\left(\mathrm{~m}, 1 \mathrm{H}, 6_{\mathrm{A}}\right) ; 8.34-8.26\left(\mathrm{~m}, 1 \mathrm{H}, 4_{\mathrm{A}}\right)$, $7.57\left(\mathrm{~m}, 2 \mathrm{H}\right.$, furyl); $7.50\left(\mathrm{~d}, J 9.02,1 \mathrm{H}, 3_{\mathrm{A}}\right) ; 6.86-6.79$ (m, $2 \mathrm{H}$, furyl); 6.56-6.53 (m, $2 \mathrm{H}$, furyl); 3.74 (d, $J$ 18.3, $\left.1 \mathrm{H}, 4_{\mathrm{B}} / 4_{\mathrm{C}}\right) ; 3.70\left(\mathrm{~d}, J 18.8,1 \mathrm{H}, 4_{\mathrm{B}} / 4_{\mathrm{C}}\right) ; 3.56(\mathrm{~d}, J 18.3,1 \mathrm{H}$, $\left.4_{\mathrm{B}}, 4_{\mathrm{C}^{\mathrm{C}}}\right) ; 3.50\left(\mathrm{~d}, J 18.8,1 \mathrm{H}, 4_{\mathrm{B}}, 4_{\mathrm{C}^{\prime}}\right) .{ }^{13} \mathrm{C}$ NMR (DMSO- $\left.d_{6}\right)$ : $\delta 168.4(\mathrm{CO}) ; 155.8\left(\mathrm{C}_{2 \mathrm{~A}}\right) ; 148.7\left(\mathrm{C}_{3 \mathrm{~B}} / \mathrm{C}_{3 \mathrm{C}}\right) ; 145.9\left(\mathrm{C}_{6 \mathrm{~A}}\right)$; 145.5 (furyl); 145.5 (furyl); $145.0\left(\mathrm{C}_{3 \mathrm{~B}} / \mathrm{C}_{3 \mathrm{C}}\right) ; 143.5\left(\mathrm{C}_{4 \mathrm{~A}}\right)$; 142.4 (furyl); 142.3 (furyl); 139.6 (furyl); 139.5 (furyl); $123.5\left(\mathrm{q},{ }^{1} J_{\mathrm{C}-\mathrm{F}} 286.8, \mathrm{CF}_{3-\mathrm{B}} / \mathrm{CF}_{3-\mathrm{C}}\right) ; 123.1\left(\mathrm{q},{ }^{1} J_{\mathrm{C}-\mathrm{F}} 286.1\right.$, $\left.\mathrm{CF}_{3-\mathrm{B}} / \mathrm{CF}_{3-\mathrm{C}}\right) ; 122.8\left(\mathrm{C}_{5 \mathrm{~A}}\right) ; 115.0$ (furyl); 113.5 (furyl); $109.3\left(\mathrm{C}_{3 \mathrm{~A}}\right) ; 92.2\left(\mathrm{q},{ }^{2} J_{\mathrm{C}-\mathrm{F}} 32.9, \mathrm{C}_{5 \mathrm{~B}} / \mathrm{C}_{5 \mathrm{C}}\right) ; 91.4\left(\mathrm{q},{ }^{2} J_{\mathrm{C}-\mathrm{F}}\right.$ 33.6, $\left.\mathrm{C}_{5 \mathrm{~B}} / \mathrm{C}_{5 \mathrm{C}}\right) ; 44.2\left(\mathrm{C}_{4 \mathrm{~B}} / \mathrm{C}_{4 \mathrm{C}}\right) ; 43.7\left(\mathrm{C}_{4 \mathrm{~B}} / \mathrm{C}_{4 \mathrm{C}}\right)$. Anal. Calc. for $\mathrm{C}_{22} \mathrm{H}_{15} \mathrm{~F}_{6} \mathrm{~N}_{5} \mathrm{O}_{5}(543.10)$ : C, 48.63; H, 2.78; N, $12.89 \%$. Found: C, 49.03; H, 2.96; N, 12.73\%. 
2-[3-(Thien-2-yl)-5-hydroxy-5-trifluoromethyl-4,5dihydro-1H-pyrazol-1-yl]-5-[3-(thien-2-yl)-5-hydroxy-5trifluoromethyl-4,5-dihydro-1H-pyrazol-1-yl-1-carbonyl] pyridine ( $3 i$ )

Yellow solid; yield 71,5\%; mp 190-192 ${ }^{\circ} \mathrm{C} .{ }^{1} \mathrm{H}$ NMR $\left(\mathrm{CDCl}_{3}\right): \delta 8.87-8.84\left(\mathrm{~m}, 1 \mathrm{H}, 6_{\mathrm{A}}\right) ; 8.35-8.29\left(\mathrm{~m}, 1 \mathrm{H}, 4_{\mathrm{A}}\right)$; 7.52-7.50 (m, $\left.1 \mathrm{H}, 3_{\mathrm{A}}\right) ; 7.47-7.44(\mathrm{~m}, 2 \mathrm{H}$, thienyl); 7.31$7.25(\mathrm{~m}, 2 \mathrm{H}$, thienyl); 7.12-7.08 (m, 2H, thienyl); $3.78(\mathrm{~d}$, $\left.J 18.3,1 \mathrm{H}, 4_{\mathrm{B}} / 4_{\mathrm{C}}\right) ; 3.74\left(\mathrm{~d}, J 18.3,1 \mathrm{H}, 4_{\mathrm{B}} / 4_{\mathrm{C}}\right) ; 3.62(\mathrm{~d}, J$ $\left.18.3,1 \mathrm{H}, 4_{\mathrm{B}}, / 4_{\mathrm{C}^{\prime}}\right) ; 3.55\left(\mathrm{~d}, J 18.3,1 \mathrm{H}, 4_{\mathrm{B}}, / 4_{\mathrm{C}^{\prime}}\right) .{ }^{13} \mathrm{C} \mathrm{NMR}$ $\left(\right.$ DMSO- $\left.d_{6}\right): \delta 164.1(\mathrm{CO}) ; 155.6\left(\mathrm{C}_{2 \mathrm{~A}}\right) ; 148.9\left(\mathrm{C}_{3 \mathrm{~B}} / \mathrm{C}_{3 \mathrm{C}}\right)$; $148.2\left(\mathrm{C}_{6 \mathrm{~A}}\right) ; 147.1\left(\mathrm{C}_{3 \mathrm{~B}} / \mathrm{C}_{3 \mathrm{C}}\right) ; 139.6\left(\mathrm{C}_{4 \mathrm{~A}}\right) ; 133.4$ (thienyl); 132.9 (thienyl); 131.0 (thienyl); 130.1 (thienyl); 129.4 (thienyl); 128.1 (thienyl); 128.0 (thienyl); 123.5 (q, ${ }^{1} J_{\text {C-F }}$ 286.8, $\mathrm{CF}_{3-\mathrm{B}} / \mathrm{CF}_{3-\mathrm{C}}$ ); 123.1 (q, ${ }^{1} J_{\mathrm{C}-\mathrm{F}} 286.1, \mathrm{CF}_{3-\mathrm{B}} / \mathrm{CF}_{3-\mathrm{C}}$ ); $122.6\left(\mathrm{C}_{5 \mathrm{~A}}\right) ; 108.9\left(\mathrm{C}_{3 \mathrm{~A}}\right) ; 92.6\left(\mathrm{q},{ }^{2} \mathrm{~J}_{\mathrm{C}-\mathrm{F}} 33.2, \mathrm{C}_{5 \mathrm{~B}} / \mathrm{C}_{5 \mathrm{C}}\right) ; 92.2$ $\left(\mathrm{q},{ }^{2} J_{\mathrm{C}-\mathrm{F}} 33.9, \mathrm{C}_{5 \mathrm{~B}} / \mathrm{C}_{5 \mathrm{C}}\right) ; 45.0\left(\mathrm{C}_{4 \mathrm{~B}} / \mathrm{C}_{4 \mathrm{C}}\right) ; 44.5\left(\mathrm{C}_{4 \mathrm{~B}} / \mathrm{C}_{4 \mathrm{C}}\right)$. Anal. Calc. for $\mathrm{C}_{22} \mathrm{H}_{15} \mathrm{~F}_{6} \mathrm{~N}_{5} \mathrm{O}_{3} \mathrm{~S}_{2}$ (575.05): $\mathrm{C}, 45.91 ; \mathrm{H}, 2.63 ; \mathrm{N}$, $12.17 \%$. Found: C, 46.28 ; H, 2.74; N, $12.31 \%$.

2-(3-Phenyl-5-hydroxy-5-trichloromethyl-4,5dihydro-1H-pyrazol-1-yl)-5-(3-phenyl-5-hydroxy-5trichloromethyl-4,5-dihydro-1H-pyrazol-1-yl-1-carbonyl) pyridine $(\mathbf{4 c})$

Yellow solid; yield 79\%; mp 204-206 ${ }^{\circ} \mathrm{C} .{ }^{1} \mathrm{H}$ NMR $\left(\mathrm{CDCl}_{3}\right): \delta 8.84-8.77\left(\mathrm{~m}, 1 \mathrm{H}, 6_{\mathrm{A}}\right) ; 8.32-8.25\left(\mathrm{~m}, 1 \mathrm{H}, 4_{\mathrm{A}}\right)$; 7.79-7.75 (m, 2H, Ph); 7.70-7.60 (m, 3H, Ph $\left.+3_{\mathrm{A}}\right) ; 7.45$ $(\mathrm{m}, 6 \mathrm{H}, \mathrm{Ph}) ; 4.07\left(\mathrm{~d}, J 18,1 \mathrm{H}, 4_{\mathrm{B}} / 4_{\mathrm{C}}\right) ; 4.03(\mathrm{~d}, J 18,1 \mathrm{H}$, $\left.4_{\mathrm{B}} / 4_{\mathrm{C}}\right) ; 3.75\left(\mathrm{~d}, J 18,1 \mathrm{H}, 4_{\mathrm{B}} / 4_{\mathrm{C}}\right) ; 3.69\left(\mathrm{~d}, J 18,1 \mathrm{H}, 4_{\mathrm{B}}, 4_{\mathrm{C}}\right)$. ${ }^{13} \mathrm{C}$ NMR (DMSO- $\left.d_{6}\right): \delta 166.0(\mathrm{CO}) ; 153.9\left(\mathrm{C}_{2 \mathrm{~A}}\right) ; 152.0$ $\left(\mathrm{C}_{3 \mathrm{~B}} / \mathrm{C}_{3 \mathrm{C}}\right) ; 148.1\left(\mathrm{C}_{6 \mathrm{~A}}\right) ; 140.8\left(\mathrm{C}_{3 \mathrm{~B}} / \mathrm{C}_{3 \mathrm{C}}\right) ; 130.9\left(\mathrm{C}_{4 \mathrm{~A}}\right) ; 130.6$ $(\mathrm{Ph}) ; 129.9(\mathrm{Ph}) ; 129.6(\mathrm{Ph}) ; 128.8(\mathrm{Ph}) ; 128,61(\mathrm{Ph}) ; 126.7$ $(\mathrm{Ph}) ; 126.5(\mathrm{Ph}) ; 125.6(\mathrm{Ph}) ; 111.6\left(\mathrm{C}_{5 \mathrm{~A}}\right) ; 103.4\left(\mathrm{CCl}_{3 \mathrm{~B}-\mathrm{B}} /\right.$ $\left.\mathrm{CCl}_{3-\mathrm{C}}\right) ; 103.2\left(\mathrm{CCl}_{3-\mathrm{B}} / \mathrm{CCl}_{3-\mathrm{C}}\right) ; 103.1\left(\mathrm{C}_{5 \mathrm{~B}} / \mathrm{C}_{5 \mathrm{C}}\right) ; 102.5\left(\mathrm{C}_{5 \mathrm{~B}} /\right.$ $\left.\mathrm{C}_{5 \mathrm{C}}\right) ; 79.0\left(\mathrm{C}_{3 \mathrm{~A}}\right) ; 46.6\left(\mathrm{C}_{4 \mathrm{~B}} / \mathrm{C}_{4 \mathrm{C}}\right) ; 46.6\left(\mathrm{C}_{4 \mathrm{~B}} / \mathrm{C}_{4 \mathrm{C}}\right)$. Anal. Calc. for $\mathrm{C}_{26} \mathrm{H}_{19} \mathrm{Cl}_{6} \mathrm{~N}_{5} \mathrm{O}_{3}(662.18): \mathrm{C}, 47.16 ; \mathrm{H}, 2.89 ; \mathrm{N}, 10.58 \%$. Found: C, 47.35; H, 2.79 ; N, 10.59\%.

2-[3-(4-Methoxyphenyl)-5-hydroxy-5-trichloromethyl4,5-dihydro-1H-pyrazol-1-yl]-5-[3-(4-methoxyphenyl)-5hydroxy-5-trichloromethyl-4,5-dihydro-1H-pyrazol-1-yl1-carbonyl]pyridine (4d)

Orange solid; yield 62\%; mp 199-201 ${ }^{\circ} \mathrm{C} .{ }^{1} \mathrm{H}$ NMR $\left(\mathrm{CDCl}_{3}\right): \delta 8.84-8.77\left(\mathrm{~m}, 1 \mathrm{H}, 6_{\mathrm{A}}\right) ; 8.31-8.25\left(\mathrm{~m}, 1 \mathrm{H}, 4_{\mathrm{A}}\right) ; 7.86-$ $7.81\left(\mathrm{~m}, 1 \mathrm{H}, 3_{\mathrm{A}}\right) ; 7.70-7.51(\mathrm{~m}, 4 \mathrm{H}, \mathrm{Ph}) ; 7.32-7.20(\mathrm{~m}, 4 \mathrm{H}$, $\mathrm{Ph}) ; 4.05\left(\mathrm{~d}, J 18,1 \mathrm{H}, 4_{\mathrm{B}} / 4_{\mathrm{C}}\right) ; 4.00\left(\mathrm{~d}, J 18,1 \mathrm{H}, 4_{\mathrm{B}} / 4_{\mathrm{C}}\right) ; 3.73$ $\left(\mathrm{d}, J 18,1 \mathrm{H}, 4_{\mathrm{B}} / 4_{\mathrm{C}}\right) ; 3.67\left(\mathrm{~d}, J 18,1 \mathrm{H}, 4_{\mathrm{B}} / 4_{\mathrm{C}}\right) ; 2.41\left(\mathrm{OCH}_{3-\mathrm{B}} /\right.$ $\left.\mathrm{OCH}_{3-\mathrm{C}}\right) ; 2.39\left(\mathrm{OCH}_{3-\mathrm{B}} / \mathrm{OCH}_{3-\mathrm{C}}\right) \cdot{ }^{13} \mathrm{C}$ NMR (DMSO- $\left.d_{6}\right): \delta$ 184.6 (CO); $180.1\left(\mathrm{C}_{2 \mathrm{~A}}\right) ; 154.0\left(\mathrm{C}_{3 \mathrm{~B}} / \mathrm{C}_{3 \mathrm{C}}\right) ; 144.5\left(\mathrm{C}_{6 \mathrm{~A}}\right) ; 140.9$ $\left(\mathrm{C}_{3 \mathrm{~B}} / \mathrm{C}_{3 \mathrm{C}}\right) ; 140.6\left(\mathrm{C}_{4 \mathrm{~A}}\right) ; 129.6(\mathrm{Ph}) ; 129.3(\mathrm{Ph}) ; 129.1(\mathrm{Ph})$; $129.0(\mathrm{Ph}) ; 128.6(\mathrm{Ph}) ; 127.3(\mathrm{Ph}) ; 126.8(\mathrm{Ph}) ; 126.6(\mathrm{Ph})$;
$110.7\left(\mathrm{C}_{5 \mathrm{~A}}\right) ; 104.1\left(\mathrm{CCl}_{3-\mathrm{B}} / \mathrm{CCl}_{3-\mathrm{C}}\right) ; 103.4\left(\mathrm{CCl}_{3-\mathrm{B}} / \mathrm{CCl}_{3-\mathrm{C}}\right)$; $103.0\left(\mathrm{C}_{5 \mathrm{~B}} / \mathrm{C}_{5 \mathrm{C}}\right) ; 102.4\left(\mathrm{C}_{5 \mathrm{~B}} / \mathrm{C}_{5 \mathrm{C}}\right) ; 79.1\left(\mathrm{C}_{3 \mathrm{~A}}\right) ; 46.6\left(\mathrm{C}_{4 \mathrm{~B}} /\right.$ $\left.\mathrm{C}_{4 \mathrm{C}}\right) ; 46.0\left(\mathrm{C}_{4 \mathrm{~B}} / \mathrm{C}_{4 \mathrm{C}}\right) ; 21.1\left(\mathrm{OCH}_{3-\mathrm{B}} / \mathrm{OCH}_{3-\mathrm{C}}\right) ; 20.9\left(\mathrm{OCH}_{3-\mathrm{B}} /\right.$ $\mathrm{OCH}_{3-\mathrm{C}}$ ). Anal. Calc. for $\mathrm{C}_{28} \mathrm{H}_{23} \mathrm{Cl}_{6} \mathrm{~N}_{5} \mathrm{O}_{5}(722.23): \mathrm{C}, 46.56 ; \mathrm{H}$, 3.21 ; N, 9.70\%. Found: C,46.32; H, 3.62; N, 9.53\%.

2-[3-(4-Nitrophenyl)-5-hydroxy-5-trichloromethyl4,5-dihydro-1H-pyrazol-1-yl]-5-[3-(4-nitrophenyl)-5hydroxy-5-trichloromethyl-4,5-dihydro-1H-pyrazol-1-yl1-carbonyl]pyridine (4e)

Yellow solid; yield 80\%; mp 211-213 ${ }^{\circ} \mathrm{C} .{ }^{1} \mathrm{H}$ NMR $\left(\mathrm{CDCl}_{3}\right): \delta 8.82-8.77\left(\mathrm{~m}, 1 \mathrm{H}, 6_{\mathrm{A}}\right) ; 8.34-8.26(\mathrm{~m}, 5 \mathrm{H}, \mathrm{Ph}+$ $\left.4_{\mathrm{A}}\right)$; 7.96-7.91 (m, 2H, Ph), 7.82-7.78 (m, 2H, Ph); 7.70$7.64\left(\mathrm{~m}, 1 \mathrm{H}, 3_{\mathrm{A}}\right) ; 4.12\left(\mathrm{~d}, J 18,1 \mathrm{H}, 4_{\mathrm{B}} / 4_{\mathrm{C}}\right) ; 4.08(\mathrm{~d}, J 18$, $\left.1 \mathrm{H}, 4_{\mathrm{B}} / 4_{\mathrm{C}}\right) ; 3.78\left(\mathrm{~d}, J 18,1 \mathrm{H}, 4_{\mathrm{B}} / 4_{\mathrm{C}}\right) ; 3.74(\mathrm{~d}, J 18,1 \mathrm{H}$, $4_{\mathrm{B}}, 4_{\mathrm{C}}$ ) ${ }^{13} \mathrm{C}$ NMR (DMSO- $\left.d_{6}\right): \delta 166.6(\mathrm{CO}) ; 157.5\left(\mathrm{C}_{2 \mathrm{~A}}\right)$; $151.5\left(\mathrm{C}_{3 \mathrm{~B}} / \mathrm{C}_{3 \mathrm{C}}\right) ; 148.3\left(\mathrm{C}_{6 \mathrm{~A}}\right) ; 148.0\left(\mathrm{C}_{3 \mathrm{~B}} / \mathrm{C}_{3 \mathrm{C}}\right) ; 140.9(\mathrm{Ph})$; $140.1(\mathrm{Ph}) ; 138.5(\mathrm{Ph}) ; 136.1\left(\mathrm{C}_{4 \mathrm{~A}}\right) ; 135.7(\mathrm{Ph}) ; 127.9(\mathrm{Ph})$; $127.5(\mathrm{Ph}) ; 126.3(\mathrm{Ph}) ; 123.9(\mathrm{Ph}) ; 110.8\left(\mathrm{C}_{5 \mathrm{~A}}\right) ; 103.9$ $\left(\mathrm{CCl}_{3-\mathrm{B}} / \mathrm{CCl}_{3-\mathrm{C}}\right) ; 103.5\left(\mathrm{CCl}_{3-\mathrm{B}} / \mathrm{CCl}_{3-\mathrm{C}}\right) ; 103.2\left(\mathrm{C}_{5 \mathrm{~B}} / \mathrm{C}_{5 \mathrm{C}}\right)$; $103.0\left(\mathrm{C}_{5 \mathrm{~B}} / \mathrm{C}_{5 \mathrm{C}}\right) ; 79.1\left(\mathrm{C}_{3 \mathrm{~A}}\right) ; 46.6\left(\mathrm{C}_{4 \mathrm{~B}} / \mathrm{C}_{4 \mathrm{C}}\right) ; 46.5\left(\mathrm{C}_{4 \mathrm{~B}} / \mathrm{C}_{4 \mathrm{C}}\right)$. Anal. Calc. for $\mathrm{C}_{26} \mathrm{H}_{17} \mathrm{Cl}_{6} \mathrm{~N}_{7} \mathrm{O}_{7}$ (752.17): C, 41.52; $\mathrm{H}, 2.28$; $\mathrm{N}, 13.04 \%$. Found: C, 41.62; H, 2.21; N, $12.94 \%$.

2-[3-(4,4'-Biphenyl)-5-hydroxy-5-trichloromethyl4,5-dihydro-1H-pyrazol-1-yl]-5-[3-(4,4'-biphenyl)-5hydroxy-5-trichloromethyl-4,5-dihydro-1H-pyrazol-1-yl1-carbonyl]pyridine $(\mathbf{4 f})$

Yellow solid; yield 85\%; mp 202-204 ${ }^{\circ} \mathrm{C} .{ }^{1} \mathrm{H}$ NMR (DMSO- $d_{6}$ ): $\delta 8.76-8.69\left(\mathrm{~m}, 1 \mathrm{H}, 6_{\mathrm{A}}\right) ; 8.28-8.26(\mathrm{~m}, 1 \mathrm{H}$, $\left.4_{\mathrm{A}}\right) ; 7.97\left(\mathrm{~d}, J\right.$ 9.02, 1H, $\left.3_{\mathrm{A}}\right) ; 7.87-7.67(\mathrm{~m}, 12 \mathrm{H}, \mathrm{BiPh})$; 7.52-7.47 (m, 4H, BiPh); 7.43-7.39 (m, 2H, BiPh); 4.13 $\left(\mathrm{d}, J 18,1 \mathrm{H}, 4_{\mathrm{B}} / 4_{\mathrm{C}}\right) ; 4.11\left(\mathrm{~d}, J 19,1 \mathrm{H}, 4_{\mathrm{B}} / 4_{\mathrm{C}}\right) ; 3.94(\mathrm{~d}, J 18$, $\left.1 \mathrm{H}, 4_{\mathrm{B}} / 4_{\mathrm{C}}\right) ; 3.90\left(\mathrm{~d}, J 19,1 \mathrm{H}, 4_{\mathrm{B}} / 4_{\mathrm{C}}\right) \cdot{ }^{13} \mathrm{C}$ NMR (DMSO- $\left.d_{6}\right)$ : $\delta 166.1(\mathrm{CO}) ; 153.6\left(\mathrm{C}_{2 \mathrm{~A}}\right) ; 151.6\left(\mathrm{C}_{3 \mathrm{~B}} / \mathrm{C}_{3 \mathrm{C}}\right) ; 147.9\left(\mathrm{C}_{6 \mathrm{~A}}\right)$; $142.3\left(\mathrm{C}_{3 \mathrm{~B}} / \mathrm{C}_{3 \mathrm{C}}\right) ; 140.7(\mathrm{Ph}) ; 140.3(\mathrm{Ph}) ; 139.2(\mathrm{Ph}) ; 138.8$ $\left(\mathrm{C}_{4 \mathrm{~A}}\right) ; 128.7(\mathrm{Ph}) ; 128.6(\mathrm{Ph}) ; 128.4(\mathrm{Ph}) ; 127.7(\mathrm{Ph}) ; 127.3$ $(\mathrm{Ph}) ; 127.3(\mathrm{Ph}) ; 127.1(\mathrm{Ph}) ; 126.9(\mathrm{Ph}) ; 126.8(\mathrm{Ph}) ; 126.6$ $(\mathrm{Ph}) ; 126.4(\mathrm{Ph}) ; 126.3(\mathrm{Ph}) ; 126.0(\mathrm{Ph}) ; 111.4\left(\mathrm{C}_{5 \mathrm{~A}}\right) ; 103.3$ $\left(\mathrm{CCl}_{3-\mathrm{B}} / \mathrm{CCl}_{3-\mathrm{C}}\right) ; 103.2\left(\mathrm{CCl}_{3-\mathrm{B}} / \mathrm{CCl}_{3-\mathrm{C}}\right) ; 103.0\left(\mathrm{C}_{5 \mathrm{~B}} / \mathrm{C}_{5 \mathrm{C}}\right)$; $102.5\left(\mathrm{C}_{5 \mathrm{~B}} / \mathrm{C}_{5 \mathrm{C}}\right) ; 78.9\left(\mathrm{C}_{3 \mathrm{~A}}\right) ; 46.6\left(\mathrm{C}_{4 \mathrm{~B}} / \mathrm{C}_{4 \mathrm{C}}\right) ; 46.5\left(\mathrm{C}_{4 \mathrm{~B}} / \mathrm{C}_{4 \mathrm{C}}\right)$. Anal. Calc. for $\mathrm{C}_{38} \mathrm{H}_{27} \mathrm{Cl}_{6} \mathrm{~N}_{5} \mathrm{O}_{3}$ (814.37): C, 56.04; H, 3.34; $\mathrm{N}, 8.60 \%$. Found: C,56.45; H, 3.51; N, 8.45\%.

General procedure for the synthesis of 2-[3-alkyl(aryl/ heteroaryl)-5-trihalomethyl-1H-pyrazol-1-yl]-5-[3alkyl(aryl/heteroaryl)-5-trihalomethyl-1H-pyrazol-1-yl1-carbonyl] pyridine (5a-c, $5 \boldsymbol{h}$ and $\mathbf{6 c})$

A solution of 2-(5-hydroxy-pyrazol-1-yl)-5-(5-hydroxypyrazol-1-yl)-pyridine (3a-c, $\mathbf{3 h}$ and $\mathbf{4 c})(2.8 \mathrm{mmol})$ and 
pyridine $(37.1 \mathrm{mmol}, 3 \mathrm{~mL})$ in $50 \mathrm{~mL}$ of benzene was cooled to $0{ }^{\circ} \mathrm{C}$ and thionyl chloride ( $16.8 \mathrm{mmol}, 1.22 \mathrm{~mL}$ ) diluted in $25 \mathrm{~mL}$ of benzene was added dropwise over $10 \mathrm{~min}$. The solution was stirred for an additional $30 \mathrm{~min}$, during which time the temperature was allowed to rise to $20^{\circ} \mathrm{C}$. The mixture was then heated under reflux (bath temperature $80^{\circ} \mathrm{C}$ ) for $1 \mathrm{~h}$ and filtered to remove the pyridine hydrochloride at room temperature. The solution was washed twice with water and dried over sodium sulfate. Evaporation of the solvent left a solid which was recrystallized from ethanol.

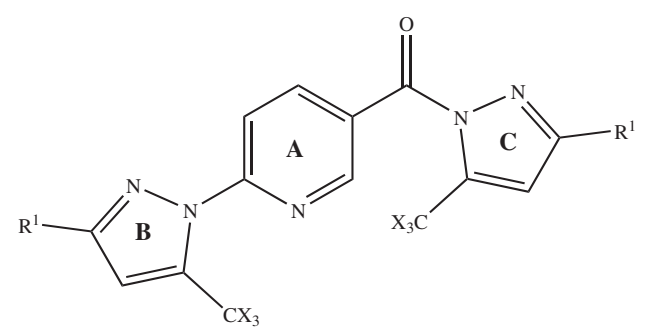

Figure 5.

2-(5-Trifluoromethyl-1H-pyrazol-1-yl)-5-(5-trifluoromethyl1H-pyrazol-1-yl-1-carbonyl) pyridine (5a)

White solid; yield 70\%; mp 104-106 ${ }^{\circ} \mathrm{C} .{ }^{1} \mathrm{H}$ NMR $\left(\mathrm{CDCl}_{3}\right): \delta 9.19-9.18\left(\mathrm{~m}, 1 \mathrm{H}, 6_{\mathrm{A}}\right) ; 8.55-8.52\left(\mathrm{~m}, 1 \mathrm{H}, 4_{\mathrm{A}}\right)$; $8.07\left(\mathrm{~d}, J 8.5,1 \mathrm{H}, 3_{\mathrm{A}}\right) ; 7.83-7.79\left(\mathrm{~m}, 2 \mathrm{H}, 4_{\mathrm{B}}\right.$ and $\left.4_{\mathrm{C}}\right) ; 7.01$ $\left(\mathrm{s}, 1 \mathrm{H}, 3_{\mathrm{B}} / 3_{\mathrm{C}}\right) ; 6.94\left(\mathrm{~s}, 1 \mathrm{H}, 3_{\mathrm{B}} / 3_{\mathrm{C}}\right) \cdot{ }^{13} \mathrm{C}$ NMR (DMSO- $\left.d_{6}\right)$ : $\delta 163.1(\mathrm{CO}) ; 153.6\left(\mathrm{C}_{2 \mathrm{~A}}\right) ; 151.5\left(\mathrm{C}_{3 \mathrm{~B}} / \mathrm{C}_{3 \mathrm{C}}\right) ; 142.2\left(\mathrm{C}_{6 \mathrm{~A}}\right)$; $141.8\left(\mathrm{C}_{3 \mathrm{~B}} / \mathrm{C}_{3 \mathrm{C}}\right) ; 141.1\left(\mathrm{C}_{4 \mathrm{~A}}\right) ; 135.3\left(\mathrm{q},{ }^{2} \mathrm{~J}_{\mathrm{C}-\mathrm{F}} 42.4, \mathrm{C}_{5 \mathrm{~B}} / \mathrm{C}_{5 \mathrm{C}}\right)$; $133.1\left(\mathrm{q},{ }^{2} J_{\mathrm{C}-\mathrm{F}} 41.7, \mathrm{C}_{5 \mathrm{~B}} / \mathrm{C}_{5 \mathrm{C}}\right) ; 125.9\left(\mathrm{C}_{5 \mathrm{~A}}\right) ; 119.8\left(\mathrm{q},{ }^{1} J_{\mathrm{C}-\mathrm{F}}\right.$ 268.5, $\left.\mathrm{CF}_{3-\mathrm{B}} / \mathrm{CF}_{3-\mathrm{C}}\right) ; 119.3\left(\mathrm{q},{ }^{1} J_{\mathrm{C}-\mathrm{F}} 269.3, \mathrm{CF}_{3-\mathrm{B}} / \mathrm{CF}_{3-\mathrm{C}}\right.$ ); $114.5\left(\mathrm{C}_{3 \mathrm{~A}}\right) ; 113.7\left(\mathrm{C}_{4 \mathrm{~B}} / \mathrm{C}_{4 \mathrm{C}}\right) ; 112.6\left(\mathrm{C}_{4 \mathrm{~B}} / \mathrm{C}_{4 \mathrm{C}}\right)$. Anal. Calc. for $\mathrm{C}_{14} \mathrm{H}_{7} \mathrm{~F}_{6} \mathrm{~N}_{5} \mathrm{O}(375.23): \mathrm{C}, 44.81 ; \mathrm{H}, 1.88 ; \mathrm{N}, 18.66 \%$. Found: C, 44.52; H, 1.60; N, 18.25\%.

2-(3-Methyl-5-trifluoromethyl-1H-pyrazol-1-yl)-5-(3methyl-5-trifluoromethyl-1H-pyrazol-1-yl-1-carbonyl) pyridine $(\mathbf{5 b})$

White solid; yield 78\%; mp 94-96 ${ }^{\circ} \mathrm{C} .{ }^{1} \mathrm{H} \mathrm{NMR}\left(\mathrm{CDCl}_{3}\right)$ : $\delta 9.165-9.161\left(\mathrm{~m}, 1 \mathrm{H}, 6_{\mathrm{A}}\right) ; 8.50-8.48\left(\mathrm{~m}, 1 \mathrm{H}, 4_{\mathrm{A}}\right) ; 8.00(\mathrm{~d}, J$ $\left.8.5,1 \mathrm{H}, 3_{\mathrm{A}}\right) ; 6.81\left(\mathrm{~s}, 1 \mathrm{H}, 4_{\mathrm{B}} / 4_{\mathrm{C}}\right) ; 6.73\left(\mathrm{~s}, 1 \mathrm{H}, 4_{\mathrm{B}} / 4_{\mathrm{C}}\right) ; 2.39$ (s, 3H, $\mathrm{CH}_{3-\mathrm{B}} / \mathrm{CH}_{3-\mathrm{C}}$ ); 2.37 (s, $\left.3 \mathrm{H}, \mathrm{CH}_{3-\mathrm{B}} / \mathrm{CH}_{3-\mathrm{C}}\right) .{ }^{13} \mathrm{C} \mathrm{NMR}$ $\left(\right.$ DMSO- $\left.d_{6}\right): \delta 162.9(\mathrm{CO}) ; 153.5\left(\mathrm{C}_{2 \mathrm{~A}}\right) ; 152.2\left(\mathrm{C}_{3 \mathrm{~B}} / \mathrm{C}_{3 \mathrm{C}}\right)$; $151.5\left(\mathrm{C}_{6 \mathrm{~A}}\right) ; 150.8\left(\mathrm{C}_{3 \mathrm{~B}} / \mathrm{C}_{3 \mathrm{C}}\right) ; 141.6\left(\mathrm{C}_{4 \mathrm{~A}}\right) ; 135.8\left(\mathrm{q},{ }^{2} J_{\mathrm{C}-\mathrm{F}}\right.$ $\left.41.7, \mathrm{C}_{5 \mathrm{~B}} / \mathrm{C}_{5 \mathrm{C}}\right) ; 133.6\left(\mathrm{q},{ }^{2} J_{\mathrm{C}-\mathrm{F}} 40.9, \mathrm{C}_{5 \mathrm{~B}} / \mathrm{C}_{5 \mathrm{C}}\right) ; 125.8\left(\mathrm{C}_{5 \mathrm{~A}}\right)$; $119.9\left(\mathrm{q},{ }^{1} J_{\mathrm{C}-\mathrm{F}} 268.5, \mathrm{CF}_{3-\mathrm{B}} / \mathrm{CF}_{3-\mathrm{C}}\right) ; 119.4\left(\mathrm{q},{ }^{1} J_{\mathrm{C}-\mathrm{F}} 269.3\right.$, $\left.\mathrm{CF}_{3-\mathrm{B}} / \mathrm{CF}_{3-\mathrm{C}}\right) ; 114.3\left(\mathrm{C}_{3 \mathrm{~A}}\right) ; 114.2\left(\mathrm{C}_{4 \mathrm{~B}} / \mathrm{C}_{4 \mathrm{C}}\right) ; 112.7\left(\mathrm{C}_{4 \mathrm{~B}} /\right.$ $\left.\mathrm{C}_{4 \mathrm{C}}\right) ; 13.5\left(\mathrm{CH}_{3-\mathrm{B}} / \mathrm{CH}_{3-\mathrm{C}}\right) ; 13.4\left(\mathrm{CH}_{3-\mathrm{B}} / \mathrm{CH}_{3-\mathrm{C}}\right)$. Anal. Calc. for $\mathrm{C}_{16} \mathrm{H}_{11} \mathrm{~F}_{6} \mathrm{~N}_{5} \mathrm{O}$ (403.28): C, 47.65; H, 2.75; N, $17.37 \%$. Found: C, 47.38; H, 2.82; N, 16.97\%.
2-(3-Phenyl-5-trifluoromethyl-1H-pyrazol-1-yl)-5-(3methyl-5-trifluoromethyl-1H-pyrazol-1-yl-1-carbonyl) pyridine $(5 \mathrm{c})$

White solid; yield $80 \%$; mp 143-145 ${ }^{\circ} \mathrm{C} .{ }^{1} \mathrm{H}$ NMR $\left(\mathrm{CDCl}_{3}\right): \delta 9.27-9.26\left(\mathrm{~m}, 1 \mathrm{H}, 6_{\mathrm{A}}\right) ; 8.66-8.61\left(\mathrm{~m}, 1 \mathrm{H}, 4_{\mathrm{A}}\right)$; $8.22\left(\mathrm{~d}, J 9,1 \mathrm{H}, 3_{\mathrm{A}}\right) ; 7.94-7.82(\mathrm{~m}, 4 \mathrm{H}, \mathrm{Ph}) ; 7.48-7.45(\mathrm{~m}$, $6 \mathrm{H}, \mathrm{Ph}) ; 7.34\left(\mathrm{~s}, 1 \mathrm{H}, 4_{\mathrm{B}} / 4_{\mathrm{C}}\right) ; 7.26\left(\mathrm{~s}, 1 \mathrm{H}, 4_{\mathrm{B}} / 4_{\mathrm{C}}\right) \cdot{ }^{13} \mathrm{C} \mathrm{NMR}$ $\left(\right.$ DMSO- $\left.d_{6}\right): \delta 162.9(\mathrm{CO}) ; 153.6\left(\mathrm{C}_{2 \mathrm{~A}}\right) ; 153.4\left(\mathrm{C}_{3 \mathrm{~B}} / \mathrm{C}_{3 \mathrm{C}}\right)$; $152.4\left(\mathrm{C}_{6 \mathrm{~A}}\right) ; 151.4\left(\mathrm{C}_{3 \mathrm{~B}} / \mathrm{C}_{3 \mathrm{C}}\right) ; 141.8\left(\mathrm{C}_{4 \mathrm{~A}}\right) ; 136.2\left(\mathrm{q},{ }^{2} J_{\mathrm{C}-\mathrm{F}}\right.$ $\left.41.6, \mathrm{C}_{5 \mathrm{~B}} / \mathrm{C}_{5 \mathrm{C}}\right) ; 134.1\left(\mathrm{q},{ }^{2} J_{\mathrm{C}-\mathrm{F}} 40.9, \mathrm{C}_{5 \mathrm{~B}} / \mathrm{C}_{5 \mathrm{C}}\right) ; 130.8(\mathrm{Ph})$; $130.0(\mathrm{Ph}) ; 129.8(\mathrm{Ph}) ; 129.2(\mathrm{Ph}) ; 128.9(\mathrm{Ph}) ; 128.8$ $(\mathrm{Ph}) ; 126.2(\mathrm{Ph}) ; 125.9(\mathrm{Ph}) ; 125.7\left(\mathrm{C}_{5 \mathrm{~A}}\right) ; 119.6\left(\mathrm{q},{ }^{1} J_{\mathrm{C}-\mathrm{F}}\right.$ 268.4, $\mathrm{CF}_{3-\mathrm{B}} / \mathrm{CF}_{3-\mathrm{C}}$ ); $119.1\left(\mathrm{q},{ }^{1} J_{\mathrm{C}-\mathrm{F}} 269.1, \mathrm{CF}_{3-\mathrm{B}} / \mathrm{CF}_{3-\mathrm{C}}\right)$; 114.2 $\left(\mathrm{C}_{3 \mathrm{~A}}\right) ; 111.3\left(\mathrm{C}_{4 \mathrm{~B}} / \mathrm{C}_{4 \mathrm{C}}\right) ; 110.0\left(\mathrm{C}_{4 \mathrm{~B}} / \mathrm{C}_{4 \mathrm{C}}\right)$. Anal. Calc. for $\mathrm{C}_{26} \mathrm{H}_{15} \mathrm{~F}_{6} \mathrm{~N}_{5} \mathrm{O}$ (527.42): $\mathrm{C}, 59.21 ; \mathrm{H}, 2.87 ; \mathrm{N}, 13.28 \%$. Found: C, 59.10; H, 2.79; N, 13.14\%.

2-(3-Fur-2-yl-5-trifluoromethyl-1H-pyrazol-1-yl)-5-(3fur-2-yl-5-trifluoromethyl-1H-pyrazol-1-yl-1-carbonyl) pyridine (5h)

Brown solid; yield $86 \%$; mp 127-129 ${ }^{\circ} \mathrm{C} .{ }^{1} \mathrm{H}$ NMR $\left(\mathrm{CDCl}_{3}\right): \delta 9.23-9.21\left(\mathrm{~m}, 1 \mathrm{H}, 6_{\mathrm{A}}\right) ; 8.62-8.56\left(\mathrm{~m}, 1 \mathrm{H}, 4_{\mathrm{A}}\right)$; $8.15\left(\mathrm{~d}, J 8.6,1 \mathrm{H}, 3_{\mathrm{A}}\right) ; 7.55$ (s, 2H, furyl); 7.25 (s, $1 \mathrm{H}$, $\left.4_{\mathrm{B}} / 4_{\mathrm{C}}\right) ; 7.17\left(\mathrm{~s}, 1 \mathrm{H}, 4_{\mathrm{B}} / 4_{\mathrm{C}}\right) ; 6.91-6.88$ (m, 2H, furyl); 6.55$6.53\left(\mathrm{~m}, 2 \mathrm{H}\right.$, furyl). ${ }^{13} \mathrm{C}$ NMR (DMSO- $d_{6}$ ): $\delta 162.8(\mathrm{CO})$; $153.3\left(\mathrm{C}_{2 \mathrm{~A}}\right) ; 151.5\left(\mathrm{C}_{3 \mathrm{~B}} / \mathrm{C}_{3 \mathrm{C}}\right) ; 146.4$ (furyl); 146.0 (furyl); 145.4 (furyl); 145.1 (furyl); $144.0\left(\mathrm{C}_{6 \mathrm{~A}}\right) ; 143.2\left(\mathrm{C}_{3 \mathrm{~B}} / \mathrm{C}_{3 \mathrm{C}}\right)$; $141.9\left(\mathrm{C}_{4 \mathrm{~A}}\right) ; 136.0\left(\mathrm{q},{ }^{2} J_{\mathrm{C}-\mathrm{F}} 42, \mathrm{C}_{5 \mathrm{~B}} / \mathrm{C}_{5 \mathrm{C}}\right) ; 134.0\left(\mathrm{q},{ }^{2} J_{\mathrm{C}-\mathrm{F}}\right.$ $\left.41.7, \mathrm{C}_{5 \mathrm{~B}} / \mathrm{C}_{5 \mathrm{C}}\right) ; 128.2\left(\mathrm{C}_{5 \mathrm{~A}}\right) ; 119.5\left(\mathrm{q},{ }^{1} J_{\mathrm{C}-\mathrm{F}} 268.9, \mathrm{CF}_{3-\mathrm{B}} /\right.$ $\left.\mathrm{CF}_{3-\mathrm{C}}\right) ; 119.1\left(\mathrm{q},{ }^{1} J_{\mathrm{C}-\mathrm{F}} 269.6, \mathrm{CF}_{3-\mathrm{B}} / \mathrm{CF}_{3-\mathrm{C}}\right) ; 114.3\left(\mathrm{C}_{4 \mathrm{~B}} / \mathrm{C}_{4 \mathrm{C}}\right)$; 111.9 (furyl); 111.6 (furyl); 111.2 (furyl); 109.9 (furyl); $108.5\left(\mathrm{C}_{4 \mathrm{~B}} / \mathrm{C}_{4 \mathrm{C}}\right)$. Anal. Calc. for $\mathrm{C}_{22} \mathrm{H}_{11} \mathrm{~F}_{6} \mathrm{~N}_{5} \mathrm{O}_{3}(507.34)$ : C, 52.08; H, 2.19; N, 13.80\%. Found: C, 52.05; H, 2.03; $\mathrm{N}, 13.28 \%$.

2-(3-Phenyl-5-trichloromethyl-1H-pyrazol-1-yl)-5-(3phenyl-5-trichloromethyl-1H-pyrazol-1-yl-1-carbonyl) pyridine ( $6 \mathrm{c}$ )

Yellow solid; yield 64\%; mp 157-159 ${ }^{\circ} \mathrm{C} .{ }^{1} \mathrm{H}$ NMR $\left(\mathrm{CDCl}_{3}\right): \delta 9.22-9.21\left(\mathrm{~m}, 1 \mathrm{H}, 6_{\mathrm{A}}\right) ; 8.56-8.51\left(\mathrm{~m}, 1 \mathrm{H}, 4_{\mathrm{A}}\right)$; $8.04\left(\mathrm{~d}, J 9,1 \mathrm{H}, 3_{\mathrm{A}}\right) ; 7.91-7.79(\mathrm{~m}, 4 \mathrm{H}, \mathrm{Ph}) ; 7.46-7.44(\mathrm{~m}$, $6 \mathrm{H}, \mathrm{Ph}) ; 7.36\left(\mathrm{~s}, 1 \mathrm{H}, \mathrm{C}_{4 \mathrm{~B}} / \mathrm{C}_{4 \mathrm{C}}\right) ; 7.35\left(\mathrm{~s}, 1 \mathrm{H}, \mathrm{C}_{4 \mathrm{~B}} / \mathrm{C}_{4 \mathrm{C}}\right) \cdot{ }^{13} \mathrm{C}$ NMR (DMSO- $\left.d_{6}\right): \delta 164.0(\mathrm{CO}) ; 154.7\left(\mathrm{C}_{5 \mathrm{~B}} / \mathrm{C}_{5 \mathrm{C}}\right) ; 152.0$ $\left(\mathrm{C}_{3 \mathrm{~B}} / \mathrm{C}_{3 \mathrm{C}}\right) ; 151.2\left(\mathrm{C}_{2 \mathrm{~A}}\right) ; 150.8\left(\mathrm{C}_{6 \mathrm{~A}}\right) ; 148.9\left(\mathrm{C}_{3 \mathrm{~B}}\right) ; 146.5$ $\left(\mathrm{C}_{5 \mathrm{~B}} / \mathrm{C}_{5 \mathrm{C}}\right) ; 141.3\left(\mathrm{C}_{4 \mathrm{~A}}\right) ; 131.2(\mathrm{Ph}) ; 130.0(\mathrm{Ph}) ; 129.9(\mathrm{Ph})$; $129.0(\mathrm{Ph}) ; 128.8(\mathrm{Ph}) ; 127.8(\mathrm{Ph}) ; 126.2(\mathrm{Ph}) ; 125.9(\mathrm{Ph}) ;$ $118.2\left(\mathrm{C}_{5 \mathrm{~A}}\right) ; 111.4\left(\mathrm{C}_{4 \mathrm{~B}} / \mathrm{C}_{4 \mathrm{C}}\right) ; 109.3\left(\mathrm{C}_{4 \mathrm{~B}} / \mathrm{C}_{4 \mathrm{C}}\right) ; 99.9\left(\mathrm{C}_{3 \mathrm{~A}}\right)$; $86.9\left(\mathrm{CCl}_{3}\right) ; 86.2\left(\mathrm{CCl}_{3}\right)$. Anal. Calc. for $\mathrm{C}_{26} \mathrm{H}_{15} \mathrm{Cl}_{6} \mathrm{~N}_{5} \mathrm{O}$ (626.15): C, 49.87; H, 2.41; N, 11.18\%. Found: C, 50.21; $\mathrm{H}, 2.47 ; \mathrm{N}, 10.78 \%$. 


\section{References}

1. Roth, H. J.; Kleemann, A.; Beisswenger, T.; Pharmaceutical Chemistry Drug Synthesis, Ellis Harwood: Chichester, 1988.

2. Brunton, L.; Lazo, J.; Parker, K.; Goodman \& Gilman's - The Pharmacological Basis of Therapeutics, $11^{\text {th }}$ ed., Mc Graw-Hill: New York, 2006.

3. Park, D. Y.; Lee, M. J.; Kim, J. N.; Kim, T. H.; Tetrahedron Lett. 2005, 46, 8799.

4. Potts, K. T.; Usifer, D. A.; Guadelupe, A.; Abruna, H. D.; J. Am. Chem. Soc. 1987, 109, 3961.

5. Hadda. T. B.; Bosec, H. L.; Polyhedron 1988, 7, 575

6. Brien, K. A.; Garner, C. M.; Pinney, K. G.; Tetrahedron 2006, 62, 3663.

7. Jameson, D. L.; Goldsby, K. A.; J. Org. Chem. 1990, 55, 4992.

8. Gamez, P.; Steensma, R. H.; Willem, L. D.; Reedijk, J.; Inorg. Chim. Acta 2002, 333, 51.

9. Kowalczyk, R.; Skarzewski, J.; Tetrahedron 2005, 61, 623.

10. Sun, X.; Yu, Z.; Wu, S.; Xiao, W. J. Organometallics 2005, 24 , 2959.

11. Mohlala, M. S.; Guzei, I. A.; Darkwa, J.; Mapolie, S .F.; J. Mol. Catal. A: Chem. 2005, 241, 93.

12. Halcrow, M. A.; Coord. Chem. Rev. 2005, 249, 2880.

13. Elhaïk, J.; Pask, C. M.; Kilner, C. A.; Halcrow, M. A.; Tetrahedron 2007, 63, 291.

14. Singh, S. P.; Kumar, D.; Jones, B. G.; Threadgill, M. D.; J. Fluorine Chem. 1999, 94, 199.

15. Padwa, A.; J. Org. Chem. 1965, 30, 1274.

16. Lednecer, D.; Mitcher, L. A.; Organic Chemistry of Drugs Synthesis, A Wiley-Interscience Publication, John Wiley \& Sons: New York, 1997.

17. Arriba, A. F.; Gómez-Casajús, L. A.; Cavalcante, F.; Almansa, C.; García-Rafanell, J.; Eur. J. Pharmacol. 1996, 318, 341.

18. Welch, J. T.; Tetrahedron 1987, 43, 3123.

19. Effenberger, F.; Maier, R.; Schonwalder, K. H.; Ziegler, T.; Chem. Ber. 1982, 115, 2766.

20. Effenberger, F.; Schonwalder, K. H.; Chem. Ber. 1984, 117, 3270 .
21. Hojo, M.; Masuda, R.; Okada, E.; Synthesis 1986, 12, 1013.

22. Hojo, M.; Masuda, R.; Sakaguchi, S.; Takagawa, M.; Synthesis 1986, 12, 1016.

23. Siqueira, G. M.; Flores, A. F. C.; Clar, G.; Zanatta, N.; Martins, M. A. P.; Quim. Nova 1994, 17, 24.

24. Flores, A. F. C.; Brondani, S.; Zanatta, N.; Rosa, A.; Martins, M. A. P.; Tetrahedron Lett. 2002, 43, 8701.

25. Bonacorso, H. G.; Costa, M. B.; Moura, S.; Pizzuti, L.; Martins, M. A. P.; Zanatta, N.; Flores. A. F. C.; J. Fluorine Chem. 2005, 126, 1396.

26. Bonacorso, H. G.; Cechinel, C. A.; Costa, M. B.; Oliveira, M. R.; Martins, M. A. P.; Zanatta, N.; Flores. A. F. C.; J. Heterocycl. Chem. 2005, 42, 1055.

27. Martins, M. A. P.; Guarda, E.; Frizzo, C. P.; Scapin, E.; Beck, P.; Costa, A. C.; Zanatta, N.; Bonacorso, H. G.; J. Mol. Catal. A: Chem. 2007, 266, 100.

28. Bonacorso, H. G.; Oliveira, M. R.; Costa, M. B.; Silva, L. B.; Wasowski, A. D.; Zanatta, N.; Martins, M. A. P.; J. Heterocycl. Chem. 2005, 42, 631.

29. Bonacorso, H. G.; Oliveira, M. R.; Costa, M. B.; Silva, L. B.; Zanatta, N.; Martins, M. A. P.; Flores, A. F. C.; J. Braz. Chem. Soc. 2005, 16, 868.

30. Bonacorso, H. G.; Oliveira, M. R.; Costa, M. B.; Drekener, R. L.; Silva, L. B.; Zanatta, N.; Martins, M. A. P.; Heteroat. Chem. 2006, 17, 685 .

31. Bonacorso, H. G.; Wentz, A. P.; Lourega, R. V.; Cechinel, C. A.; Moraes, T. S.; Coelho, H. S.; Zanatta, N.; Martins, M. A. P.; Höerner, M.; Alves, S. H.; J. Fluorine Chem. 2006, 127, 1066.

32. Crystallographic data for compound $\mathbf{3 c}$ and $\mathbf{5 a}$, reported in this paper, have been deposited with the Cambridge Crystallographic Data Center (CCDC 686704 and 699775, respectively). Copies of the data can be obtained, free of the charge, on application to CCDC 12 Union Road, Cambridge CB2 1EZ, UK (Fax +441223-336033 or e-mail deposit@ccdc.cam.ac.uk).

Received: November 5, 2008 Web Release Date: March 6, 2009 